\title{
High-speed photometry of Gaia14aae: an eclipsing AM CVn that challenges formation models
}

\author{
M. J. Green, ${ }^{1 \star}$ T. R. Marsh, ${ }^{1}$ D. T. H. Steeghs, ${ }^{1}$ T. Kupfer,${ }^{2}$ R. P. Ashley, ${ }^{1}$ S. Bloemen, ${ }^{3}$ \\ E. Breedt, ${ }^{1,4}$ H. C. Campbell, ${ }^{4,5}$ A. Chakpor, ${ }^{6}$ C. M. Copperwheat, ${ }^{7}$ \\ V. S. Dhillon, ${ }^{8,9}$ G. Hallinan, ${ }^{10}$ L. K. Hardy, ${ }^{8}$ J. J. Hermes,${ }^{11} \dagger$ P. Kerry, ${ }^{8}$ \\ S. P. Littlefair, ${ }^{8}$ J. Milburn, ${ }^{10}$ S. G. Parsons,${ }^{8}$ N. Prasert, ${ }^{6}$ J. van Roestel, ${ }^{3}$ \\ D. I. Sahman ${ }^{8}$ and N. Singh ${ }^{10,12}$ \\ ${ }^{1}$ Astronomy and Astrophysics Group, Department of Physics, University of Warwick, Coventry CV4 7AL, UK \\ ${ }^{2}$ Division of Physics, Mathematics and Astronomy, California Institute of Technology, Pasadena, CA 91125, USA \\ ${ }^{3}$ Department of Astrophysics/IMAPP, Radboud University, PO Box 9010, NL-6500 GL Nijmegen, the Netherlands \\ ${ }^{4}$ Institute of Astronomy, University of Cambridge, Madingley Road, Cambridge CB3 OHA, UK \\ ${ }^{5}$ Department of Physics, University of Surrey, Guildford GU2 7XH, UK \\ ${ }^{6}$ National Astronomical Research Institute of Thailand (Public Organization), 260 Moo 4, T. Donkaew, A. Maerim, Chiangmai 50180, Thailand \\ ${ }^{7}$ Astrophysics Research Institute, Liverpool John Moores University, Liverpool L3 5RF, UK \\ ${ }^{8}$ Department of Physics and Astronomy, University of Sheffield, Sheffield $S 37 R H, U K$ \\ ${ }^{9}$ Instituto de Astrofísica de Canarias, E-38205 La Laguna, Tenerife, Spain \\ ${ }^{10}$ Cahill Centre for Astronomy and Astrophysics, California Institute of Technology, Pasadena, CA 91125, USA \\ ${ }^{11}$ Department of Physics and Astronomy, University of North Carolina, Chapel Hill, NC 27599-3255, USA \\ ${ }^{12}$ Jet Propulsion Laboratory, California Institute of Technology, Pasadena, CA 91109, USA
}

${ }^{\star}$ E-mail: matthew.green@warwick.ac.uk

$\dagger$ Hubble Fellow.

\begin{abstract}
AMCVn-type systems are ultracompact, hydrogen-deficient accreting binaries with degenerate or semidegenerate donors. The evolutionary history of these systems can be explored by constraining the properties of their donor stars. We present high-speed photometry of Gaia14aae, an AMCVn with a binary period of $49.7 \mathrm{~min}$ and the first AMCVn in which the central white dwarf is fully eclipsed by the donor star. Modelling of the light curves of this system allows for the most precise measurement to date of the donor mass of an AMCVn, and relies only on geometric and well-tested physical assumptions. We find a mass ratio $q=M_{2} / M_{1}=0.0287 \pm 0.0020$ and masses $M_{1}=0.87 \pm 0.02 \mathrm{M}_{\odot}$ and $M_{2}=0.0250 \pm 0.0013 \mathrm{M}_{\odot}$. We compare these properties to the three proposed channels for AMCVn formation. Our measured donor mass and radius do not fit with the contraction that is predicted for AMCVn donors descended from white dwarfs or helium stars at long orbital periods. The donor properties we measure fall in a region of parameter space in which systems evolved from hydrogen-dominated cataclysmic variables are expected, but such systems should show spectroscopic hydrogen, which is not seen in Gaia14aae. The evolutionary history of this system is therefore not clear. We consider a helium-burning star or an evolved cataclysmic variable to be the most likely progenitors, but both models require additional processes and/or fine-tuning to fit the data. Additionally, we calculate an updated ephemeris which corrects for an anomalous time measurement in the previously published ephemeris.
\end{abstract}

Key words: binaries: close-binaries: eclipsing-stars: dwarf novae-stars: individual: Gaia14aae - novae, cataclysmic variables - white dwarfs.

\section{INTRODUCTION}

AMCVn-type systems are a class of compact binaries in which white dwarfs accrete helium-dominated matter from low-mass degenerate or semidegenerate companions. Their short orbital 
periods (5-65 $\mathrm{min}$ ) and deficiency of hydrogen make them probes of extreme physics, and they will be among the first systems detected by a space-based gravitational wave interferometer (Korol et al. 2017; Nelemans 2003). Alongside the menagerie of hydrogen-dominated accreting white dwarfs (cataclysmic variables, CVs) they are laboratories of accretion physics (Kotko et al. 2012; Cannizzo \& Nelemans 2015). They experience dwarf nova outbursts, and are evolutionarily related to the double-degenerate pathway towards type Ia supernovae. They are also proposed sources of subluminous '.Ia' supernovae (Bildsten et al. 2007) and may be sources of helium novae (such as V445 Pup, Woudt et al. 2009). $\mathrm{AMCVns}$ are rare in comparison to hydrogen-rich CVs, but the number of known systems has increased dramatically in recent years due to transient surveys (e.g. Levitan et al. 2011, 2013) and follow-up surveys based on colour selection from the Sloan Digital Sky Survey (SDSS; Roelofs, Nelemans \& Groot 2007; Carter et al. 2013, 2014). Around 50 systems are now known, twice the number quoted in the most recent review (Solheim 2010). However, the space density of the AMCVn population continues to fall short of predictions based on binary population synthesis models (Carter et al. 2013).

$\mathrm{AMCVns}$ are the end point of a finely tuned evolutionary process involving either one or two common-envelope phases. As such, they provide an opportunity to calibrate models of interacting binary evolution, including the poorly understood common-envelope phase (Ivanova et al. 2013). There are three proposed channels by which AMCVn binaries may form, but the contribution of each channel to the AMCVn population is poorly constrained.

In the white dwarf channel (Paczyński 1967) and the helium-star channel (Savonije, de Kool \& van den Heuvel 1986; Iben \& Tutukov 1987), the binary passes through two common-envelope stages as each of its component stars leaves the main sequence. During these stages, the binary is surrounded by an envelope of material that extracts energy from the system, reducing the orbital period of the system to below the period minimum of hydrogen-dominated, nonmagnetic CVs. The channels differ in the nature of the secondary star following the ejection of the second common envelope; in the white dwarf channel it is left as a low-mass, degenerate or semidegenerate white dwarf, whereas in the helium-star channel, it is a non-degenerate helium-burning star. Deloye et al. (2007) and Yungelson (2008) predict that donors from both the white dwarf and helium-star channels should evolve towards complete degeneracy at periods $\gtrsim 40 \mathrm{~min}$. For periods longer than this, the predicted donor mass for both channels is almost a unique function of orbital period alone. A similar convergence is predicted for helium white dwarf donors in ultra-compact X-ray binaries, a class of object with similar evolutionary paths (Sengar et al. 2017).

These two channels are predicted by some models to dominate the formation of AMCVns at short periods $(<25 \mathrm{~min}$, Nelemans, Yungelson \& Portegies Zwart 2004). On the other hand, Shen (2015) argues that friction within the ejecta of He novae could cause all double white dwarf binaries to merge directly rather than reaching a state of stable accretion, rendering the white dwarf donor channel essentially impossible.

The third formation channel is the evolved-CV, or evolved mainsequence donor, channel (Tutukov et al. 1985; Podsiadlowski, Han \& Rappaport 2003; Goliasch \& Nelson 2015). In this channel, the donor must evolve off the main sequence at around the time of the start of mass transfer. The system then appears as a hydrogendominated $\mathrm{CV}$ in its early evolution, becoming helium-dominated as the hydrogen envelope of the donor is stripped. This channel favours the formation of longer period AMCVns (>40 min). In addition to AMCVns, this channel is predicted to produce evolved $\mathrm{CVs}$ which contain both hydrogen and helium and have periods below the CV period minimum. Several CVs in the 50-76 min period range have indeed been found which are possibly products of this channel (e.g. Augusteijn et al. 1996; Breedt et al. 2012). However, the small number of such systems compared to the number of AMCVns suggests that the channel should not be a major contributor to the AMCVn population. This is consistent with population synthesis predictions which predict the formation of only a small number of AMCVns via this channel, due to the finely tuned starting parameters and long time-scales required to remove all visible hydrogen from these systems (e.g. Goliasch \& Nelson 2015).

Population estimates for these formation channels are poorly constrained by data due to the difficulty in distinguishing the products of these channels from one another. Products of the white dwarf and helium-star channels can be distinguished by the nature of their donor stars, as these channels produce donors with different levels of degeneracy which hence occupy different regions of a mass-radius diagram (Deloye et al. 2007; Yungelson 2008). Due to the faintness of the donor star, it cannot be observed directly. For many systems, it can only be studied by indirect methods such as measuring the radial velocity of the accretor (e.g. Kupfer et al. 2016; Roelofs et al. 2006), which generally does not yield results with the required precision to distinguish between the two channels, or methods based on the superhump period (e.g. Kato \& Osaki 2013) which arise from models that have not been well tested for helium-dominated systems.

In hydrogen CVs, high-speed photometry of eclipsing systems has yielded the most precise measurements of component masses and radii while relying only on assumptions about the geometry of the system. (e.g. Savoury et al. 2011; Littlefair et al. 2014; McAllister et al. 2015). Non-magnetic CVs have a geometry consisting of several key components: the donor and accretor stars, an accretion disc around the accretor, a stream of matter passing from the donor to the accretor, and a 'bright spot' (sometimes referred to as a 'hotspot') on the edge of the accretion disc at the point of intersection with the infalling matter stream. During eclipse these components add characteristic features to the light curve which can be used to constrain the properties of the system (Cook \& Warner 1984; Wood et al. 1986).

Only three eclipsing AMCVns have been discovered to date: PTF1J1919+4815, in which just the edge of the disc and bright spot are eclipsed (Levitan et al. 2014); YZLMi (also known as SDSS J092638.71+362402.4), in which the white dwarf is partially eclipsed (Anderson et al. 2005; Copperwheat et al. 2011); and Gaia14aae (also known as ASASSN-14cn), the only known AMCVn in which the white dwarf is fully eclipsed (Campbell et al. 2015). Due to their eclipsing nature, the latter two of these systems are ideal targets for parameter studies. Copperwheat et al. (2011) used eclipse fitting with high time resolution photometry to measure the donor mass of YZ LMi. This paper does the same for Gaia14aae.

Gaia14aae has a $g$ ' magnitude of 18.3-18.6 outside of eclipse and an orbital period of $49.71 \mathrm{~min}$, putting it at the long-period end of the AM CVn distribution. Gaia14aae experienced two outbursts in 2014 June and August, but no outbursts have been recorded since then. Following the discovery of Gaia14aae, Campbell et al. (2015) used time series photometry to constrain the properties of the system. They were not able to measure the mass ratio, but were able to set a lower limit of $q>0.019$, and found corresponding minimum masses for the primary and secondary of 0.78 and $0.015 \mathrm{M}_{\odot}$ respectively.

This paper presents follow-up photometry and analysis of Gaia14aae. In Section 2, we will describe how the data were taken 
Table 1. Summary of the observations carried out for this paper.

\begin{tabular}{|c|c|c|c|c|}
\hline Observatory & Date at start (UT) & Filters & Exposure time (s) * & $\begin{array}{c}\text { Number of } \\
\text { eclipses }\end{array}$ \\
\hline \multirow[t]{6}{*}{ WHT + ULTRACAM } & 2015 Jan 14 & $u^{\prime} g^{\prime} i$ & $3(9)$ & 3 \\
\hline & 2015 Jan 15 & $u^{\prime} g^{\prime} r^{\prime}$ & $3(9)$ & 4 \\
\hline & 2015 Jan 16 & $u^{\prime} g^{\prime} r^{\prime}$ & $3(9)$ & 5 \\
\hline & 2015 Jan 17 & $u^{\prime} g^{\prime} r^{\prime}$ & $3(9)$ & 3 \\
\hline & 2015 May 23 & $u^{\prime} g^{\prime} r^{\prime}$ & $2.5(7.5)$ & 6 \\
\hline & 2015 Jun 22 & $u^{\prime} g^{\prime} r$ & $3(9)$ & 4 \\
\hline \multirow[t]{4}{*}{ TNT + ULTRASPEC } & 2016 Mar 12 & $K G 5$ & 8 & 1 \\
\hline & 2016 Mar 13 & $K G 5$ & 5 & 2 \\
\hline & 2016 Mar 14 & $K G 5$ & 5 & 3 \\
\hline & 2016 Mar 15 & $g^{\prime}$ & 5 & 2 \\
\hline \multirow[t]{3}{*}{ Hale + CHIMERA } & 2016 Aug 06 & $g^{\prime} r$ & 4 & 7 \\
\hline & 2016 Aug 07 & $g^{\prime} r^{\prime}$ & 4 & 6 \\
\hline & 2016 Aug 08 & $g^{\prime} r '$ & 4 & 6 \\
\hline TNT + ULTRASPEC & 2017 Feb 21 & $K G 5$ & 4 & 1 \\
\hline
\end{tabular}

and reduced. Section 3 will present the data. Section 4 will describe the process used to fit models to the eclipses and present the results. In Section 5, we will compare these results to modelled evolutionary tracks.

\section{OBSERVATIONS}

In this paper, we present high-speed, multicolour photometric observations of Gaia14aae, taken using the high-speed CCD cameras ULTRACAM, ULTRASPEC, and The Caltech HIgh-speed Multicolour camERA (CHIMERA). We observed 53 eclipses, each in 1-3 colour bands, spanning a time period of 25 months. A summary of observations is presented in Table 1 .

Images were taken using ULTRACAM (Dhillon et al. 2007) on the $4.2 \mathrm{~m}$ William Herschel Telescope (WHT). ULTRACAM is a three-beam camera, allowing it to record images in three colour bands simultaneously, while using frame-transfer CCDs to reduce dead time between exposures to negligible amounts $(25 \mathrm{~ms})$. Data were also collected using ULTRASPEC (Dhillon et al. 2014), a single-band photometer with a frame-transfer, electron-multiplying CCD and only $15 \mathrm{~ms}$ dead time per cycle, which is mounted on the $2.4 \mathrm{~m}$ Thai National Telescope (TNT). Most of the ULTRASPEC data were taken using a custom filter, $K G 5$, which has a broader band to allow for shorter exposure times given the smaller collecting area of this telescope (for more details on the KG5 band, see Hardy et al. 2017). A further series of eclipses were observed using CHIMERA (Harding et al. 2016), a two-band photometer which uses frametransfer, electron-multiplying CCDs to achieve $15 \mathrm{~ms}$ dead time, and is mounted on the Hale 200-inch (5.1 m) Telescope.

The images from all three instruments were reduced using the ULTRACAM reduction pipeline, described in Dhillon et al. (2007). Each of the images was bias-subtracted and divided by twilight flat-fields. The ULTRACAM and ULTRASPEC data were also dark-subtracted. The flux of Gaia14aae was then extracted using aperture photometry, dividing the flux in each frame by a comparison star (J2000 coordinates 16:11:30.53, +63:09:25.8) to remove any atmospheric transparency variations.

The $u^{\prime} g$ ' $r$ ' $i$ ' fluxes were then calibrated using the comparison star, the magnitude of which is available from SDSS $\left(m_{u^{\prime}}=17.52\right.$, $m_{g^{\prime}}=15.45, m_{r^{\prime}}=14.62$, and $\left.m_{i^{\prime}}=14.34\right)$. We ensured that this star did not show variability and we tested this calibration using several nearby comparison stars, each of which resulted in consistent calibrations. Although the $K G 5$ band can be flux-calibrated (see the appendix of Hardy et al. 2017), we did not calibrate these data because they are used for timing purposes only.

\section{PHOTOMETRY}

The complete set of photometry is shown in Figs 1-3, and Fig. 4 shows an example set of individual eclipses. Fig. 5 shows $r$ '- and $g$ '-band phase-folded eclipses.

The flux from Gaia14aae is dominated by the central white dwarf, with the accretion disc and bright spot also making measurable contributions. Due to its low temperature, the contribution of the donor is not seen. Fig. 5 shows a decomposition of the contributions of each component to the eclipse profile. The bright spot enters eclipse just as the white dwarf emerges, weakening the white dwarf's egress feature and causing an asymmetry in its eclipse shape. Some light curves (Fig. 1) also show a periodic 'hump' feature around phases -0.5 to 0 , which is commonly seen in CVs and which indicates that the light from the bright spot is preferentially emitted in a certain direction rather than being isotropic.

Gaia14aae shows the short-term variability known as 'flickering' which is commonly observed in accreting systems. This arises from the variable nature of the accretion, and is generally localized to the bright spot and the inner disc. Flickering provides a source of correlated noise that is difficult to deal with analytically, particularly as the amplitude of the variability changes throughout the cycle with the eclipse of the bright spot and inner disc (McAllister et al. 2017). In Gaia14aae, the amplitude of the flickering is similar to the depth of the bright spot eclipse, meaning that in some cycles the bright spot eclipse is completely hidden by the flickering (see some of the examples in Fig. 4). The amplitude of flickering in Gaia14aae varies significantly between epochs, with the system being particularly variable in some epochs (e.g. 2016 March, Fig. 2) and comparatively stable in others (e.g. 2016 August, Fig. 3).

Alongside this flickering, Gaia14aae shows a significant amount of variability on longer time-scales. The total flux from the system decreases by approximately 30 percent between the earliest and latest observations, with no corresponding colour change. Nights such as 2015 May 23 (Fig. 1) show a long-term variation in flux. The depths of both the bright spot eclipse and the disc eclipse 


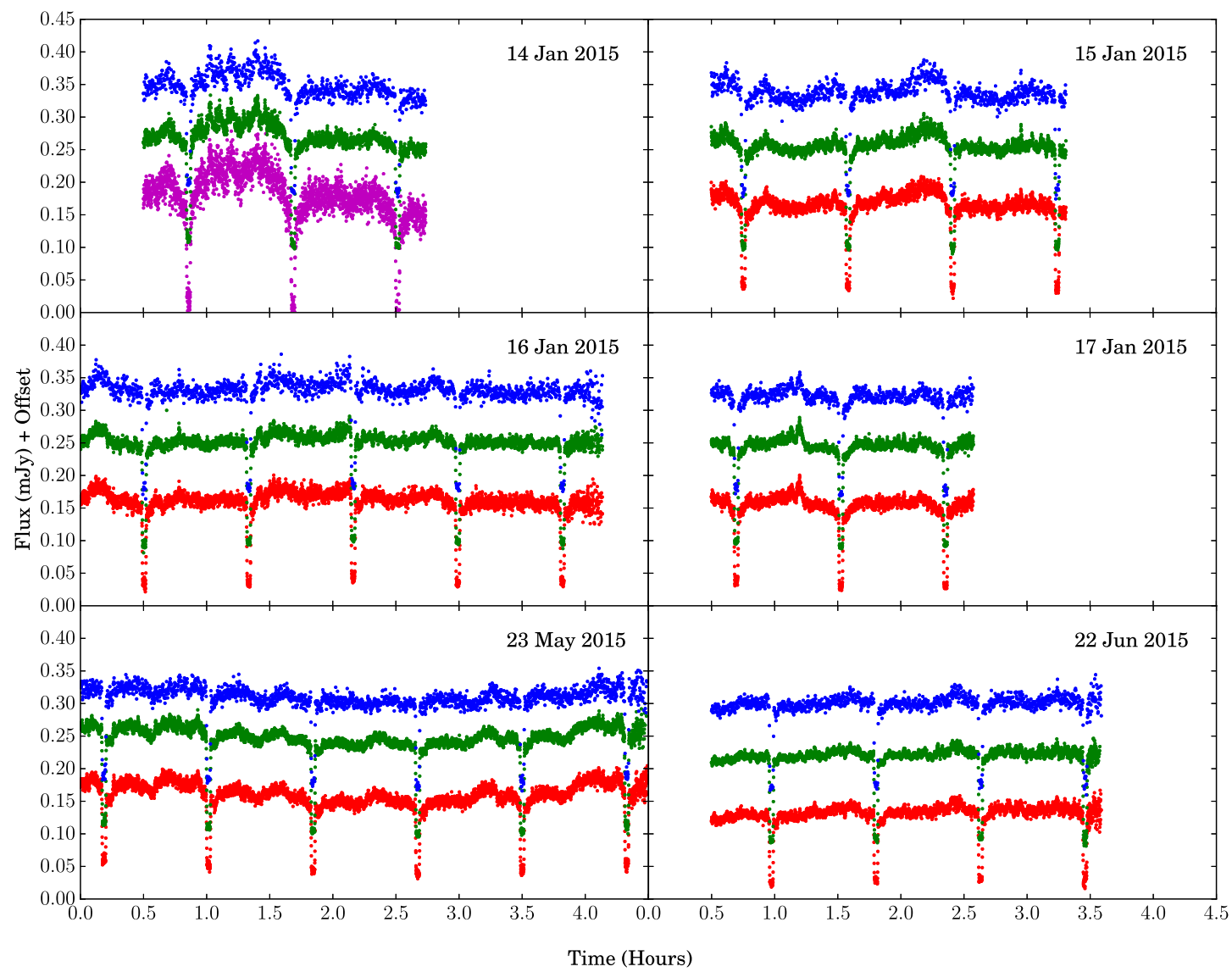

Figure 1. ULTRACAM photometry taken on the $4.2 \mathrm{~m}$ WHT in 2015. Data shown were taken in $u^{\prime}$ (blue), g' (green), $r^{\prime}$ (red), and $i$ ' (magenta). The $i^{\prime}, g^{\prime}$, and $u$ ' data have been offset in the $y$-direction by $-0.07,0.07$, and $0.15 \mathrm{mJy}$, respectively. No offset has been applied to the $r$ ' data. Data with error bars more than 3.5 times the mean (due to cloud) have been removed for clarity.

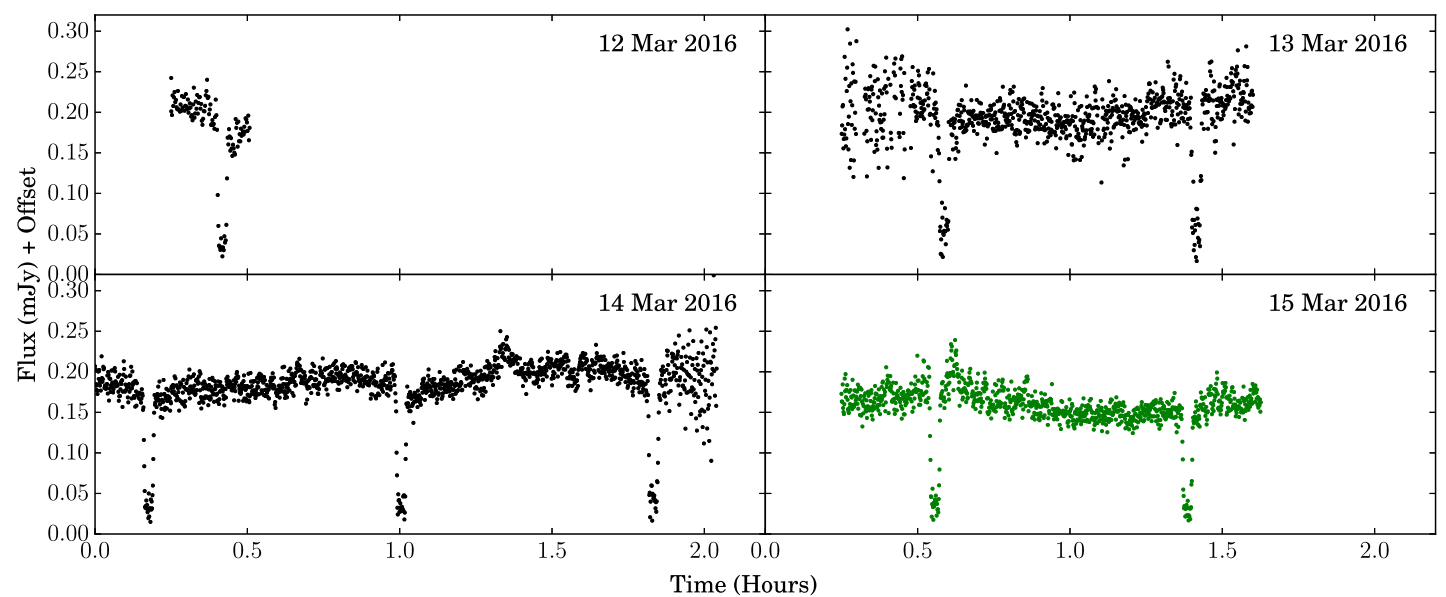

Figure 2. ULTRASPEC photometry taken on the $2.4 \mathrm{~m}$ TNT in 2016. Data were taken in $K G 5$ (black) and $g$ ' (green). Note that the scaling of the $K G 5$ data is arbitrary as those data are not flux-calibrated. Data with error bars more than 3.5 times the mean (due to cloud) have been removed for clarity.

change significantly throughout the period of observations, both being particularly prominent in 2015 January (Fig. 1) and barely visible by 2016 August (Fig. 3). The phase of the bright spot ingress also appears later in the 2016 data than in the 2015 data, which may imply an increase in the accretion disc radius. This is somewhat unexpected as the disc radius is usually expected to decrease with time during periods of quiescence (e.g. Wood et al. 1989). The depth of the white dwarf eclipse shows a significant decrease over the time period of observation (see Section 5.2 for further analysis of the white dwarf eclipse depth).

\section{LIGHT CURVE MODELLING}

The light curves contain the necessary information to derive the donor mass of the system (Cook \& Warner 1984; Wood et al. 1986), 


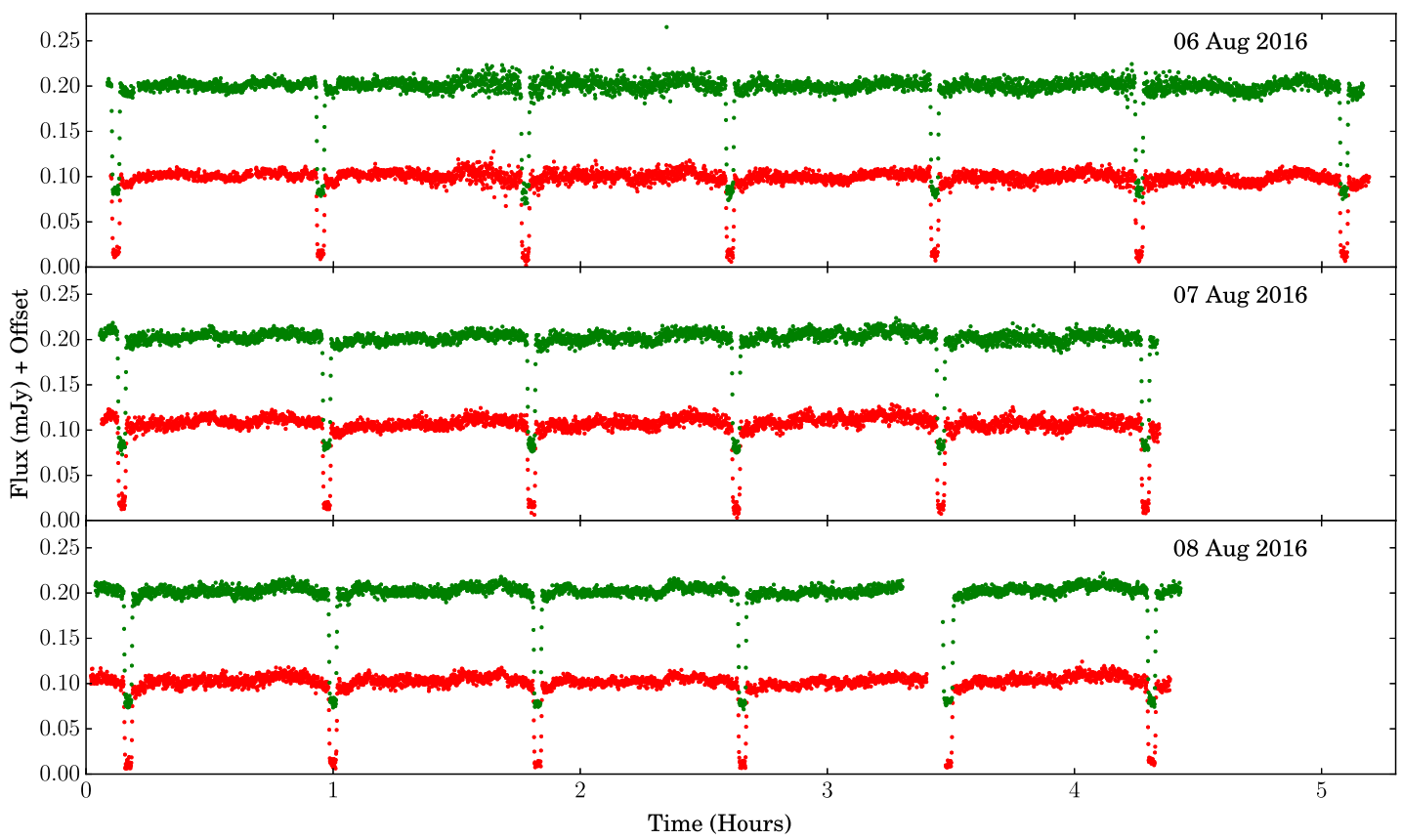

Figure 3. The CHIMERA data taken on the $5.1 \mathrm{~m}$ Hale telescope in 2016 August. These data were taken in $g$ ' (green) and $r^{\prime}$ (red). The $g$ ' data have been offset by $0.07 \mathrm{mJy}$. Data with error bars more than 3.5 times the mean (due to cloud) have been removed for clarity.

given an assumed $M-R$ relation for the central white dwarf. If the donor is assumed to be Roche lobe filling, its radius as a fraction of orbital separation, $R_{2} / a$, is a function of the mass ratio, $q$, of the two stars. The phase width of the white dwarf eclipse $\Delta \phi$ therefore depends only on $q$ and the orbital inclination $i$. For a given $\Delta \phi$, the radius of the donor (and hence $q$ ) can be increased by moving the system towards lower orbital inclinations (more face-on). A lower limit of $q$ can be found from $\Delta \phi$ by assuming $i=90^{\circ}$. Using this method, Campbell et al. (2015) found $q>0.019$ for Gaia14aae.

Lifting the degeneracy between $q$ and $i$ requires additional information, which can be found from the contact phases of the bright spot eclipse. The path of the infalling stream of matter relative to the two stars (on which the bright spot is assumed to lie) is a function of $q$. The bright spot ingress and egress phases therefore provide an additional constraint on $q$ and $i$ that serves to disentangle them.

Once $q$ and $i$ are known, the radius $R_{1}$ of the accreting white dwarf can be found from the duration of the ingress or egress of the white dwarf eclipse $\Delta w$. An assumed $M-R$ relation for the accretor can then give $M_{1}$, and hence $M_{2}$ can be found from $q$.

\subsection{MCMC modelling}

We modelled the eclipses of Gaia14aae using the package LCURVE. A description of the model can be found in Copperwheat et al. (2010, appendix), but we give a brief overview here. The code models each component of the system (white dwarf, accretion disc, bright spot, and donor star) as a grid of elements, and for each time-step computes which elements are occulted by the donor star. The white dwarf is assumed to be spherical, the disc to be $2 \mathrm{D}$ and azimuthally symmetric, and the donor to be Roche lobe filling. The bright spot is modelled as a 1D line of points, of whose light some fraction is emitted isotropically, while the remainder is 'beamed' at an angle from the system, allowing for the recreation of the hump feature in light curves. Key variables in the model are the mass ratio $q$, orbital inclination $i$, mid-time of the white dwarf eclipse $t_{0}$, orbital period $P_{\text {orb }}$, the relative temperatures of all components, the radius of the accretor, the outer radius of the disc, and the orientation, beamed fraction and angle of beaming of the bright spot. All variables are listed in Table 2. Limb-darkening of the accretor was described according to Gianninas et al. (2013) for a $13000 \mathrm{~K}, \log (g)=8.5$ white dwarf.

In order to find the optimal values and uncertainties of these parameters, this model was converged on the data using Markov Chain Monte Carlo (MCMC) code implemented by the PYTHON package EMCEE (Foreman-Mackey et al. 2013). This package implements an affine-invariant ensemble sampling algorithm (Goodman \& Weare 2010) in which the parameter space is explored by a cloud of 'walkers'. At each iteration, new positions for each walker are proposed according to the positions of other walkers in the ensemble. In this way, the number of tuning parameters is vastly reduced compared to Metropolis-Hastings algorithms. A 'scale factor' is required to tune the scale of each move; we left this at its default value of 2 . For each parameter in each fit, the best-fitting value was taken to be the median value from all chains after the burn-in phase, with $1 \sigma$ error bars taken as the standard deviation.

In order to measure changes in white dwarf flux and disc flux between eclipses, each eclipse in each colour band was first fitted separately using a shorter MCMC run. The ULTRACAM data were converged on using 50 walkers and a minimum of 20000 trials. The CHIMERA data were converged on using 200 walkers and a minimum of 5000 trials. This was sufficient to measure the relative flux of each component. Values of $q$ or $i$ based on individual eclipses have large error bars, and some eclipses are biased by flickering, by the coincidence of white dwarf egress with bright spot ingress, or show too faint a bright spot eclipse for $q$ to be well constrained. However, they are sufficient to provide a warning if the fit to the phase-folded light curve were a long way from the true value, and to compare properties of the white dwarf and disc which are independent of the bright spot parameters. 

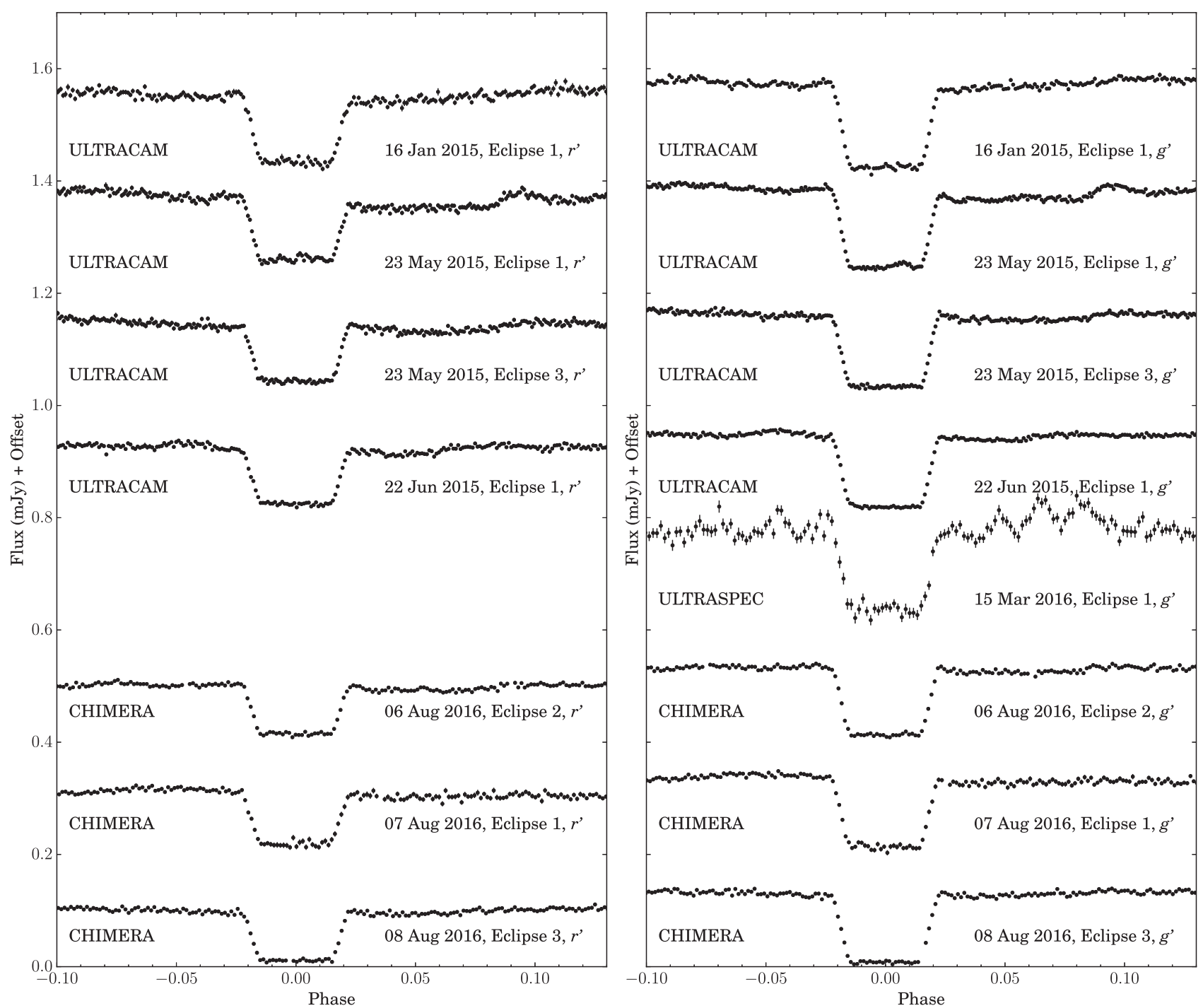

Figure 4. Example light curves of Gaia14aae, demonstrating the variability of the eclipse shape. Each light curve has been offset by $0.2 \mathrm{mJy}$ from the light curve below it.
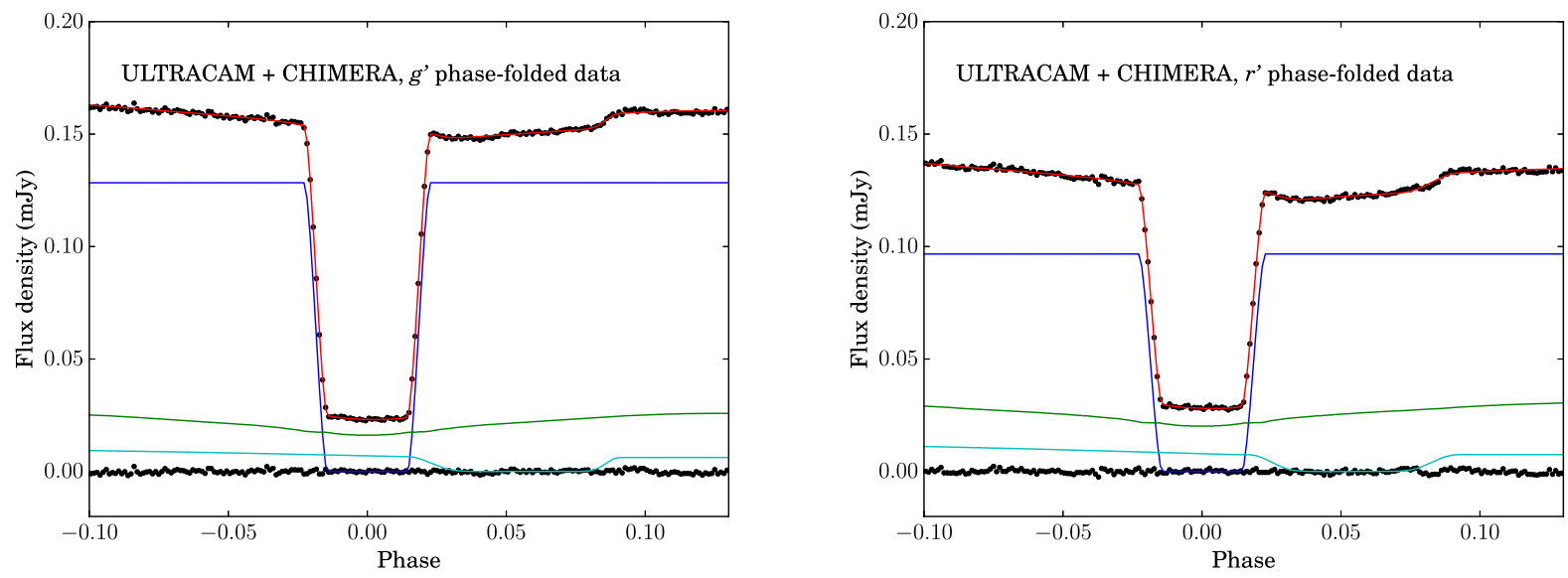

Figure 5. Phase-folded, rescaled, and averaged light curves combining both ULTRACAM and CHIMERA data, in $g$ ' and $r^{\prime}$ (left and right respectively, black points). The light curves show the consecutive eclipses of the accreting white dwarf and the bright spot. We show our best-fitting models to each (red line) and the residuals. We also show a breakdown of the light-curve contributions of each component: central white dwarf (blue), accretion disc (green), and bright spot (cyan). The donor's contribution was assumed to be negligible. There is a feature following the egress of the bright spot (phase 0.1 ) which is not described by the model, and may be due to flickering. 
Table 2. Details on variables in LCURVE and any constraints applied during the fit to the phase-folded light curves.

\begin{tabular}{ll}
\hline Variable & Description \\
\hline$t_{0}$ & Mid-time of the white dwarf eclipse \\
$q$ & Mass ratio $M_{2} / M_{1}$ \\
$\Delta i$ & Departure of inclination $i$ from that expected given $q$ and the average eclipse width $\Delta \phi=0.037$ \\
$R_{1}$ & Radius of the white dwarf \\
$R_{\text {brightspot }}$ & Radial distance of the spot from the centre of the white dwarf \\
$R_{\text {disc }}$ & Radial distance of the disc edge from the centre of the white dwarf \\
$T_{\text {whitedwarf }}$ & Effective temperatures of the components - these are really scaling factors for the flux \\
$T_{\text {brightspot }}$ & contributions and do not reflect the true temperatures of these components \\
$T_{\text {disc }}$ & Scale factor for the line of elements that make up the bright spot \\
Spot length & Angle between the line of elements of the bright spot and a tangent to the edge of the circle \\
Spot angle & Fraction of light from the bright spot which is 'beamed' in a certain direction, \\
Spot fraction & producing the observed hump in the light curves \\
& Beaming angle of the light from the bright spot in the orbital plane \\
Spot yaw &
\end{tabular}

To measure $q$ and $i$, we produced phase-folded, binned, and averaged light curves using all ULTRACAM and CHIMERA data in the $g$ ' and $r$ ' bands. The ULTRASPEC data were excluded because these data have larger error bars, they were taken during poorer observing conditions, and the system showed more than the usual amount of flickering during that run. Data in $i$ ' and $u$ ' were excluded due to the smaller number of eclipses available for both, as well as the poorer cadence and noisier data in $u$ '.

To deal with the variability in both white dwarf eclipse depth and baseline brightness, the individual eclipses were scaled based on their brightness both in-eclipse (phase 0) and after-eclipse (phases $0.1-0.5)$ so as to reduce the variability within each bin. In the model fitting described above, $q$ and $i$ are constrained primarily by the ingress and egress phases of the white dwarf and bright spot, and therefore should not be affected by changes in the flux from the system. The phases of the bright spot can change if the disc radius changes; however, as only a small change in the disc radius is seen, this is likely not to be significant compared to other sources of uncertainty (particularly the scatter in white dwarf radius discussed in Section 5.2). These light curves were converged on with 200 walkers and a minimum of 10000 trials.

From each fitted model, we found a value for $M_{2}$ based on the measured values of $q$ and $R_{1} / a$, Kepler's laws, and a white dwarf $M-R$ relation for the central white dwarf (that of P. Eggleton as quoted in Verbunt \& Rappaport 1988). The $M-R$ relation was scaled by a factor of 0.9985 , chosen as the ratio between the Eggleton radius and the stellar model radius for a DB white dwarf of temperature and mass similar to ours (Holberg \& Bergeron 2006; Bergeron et al. 2011). This scaling factor represents both the non-zero temperature of the white dwarf and the lack of hydrogen in its atmosphere. We assumed a white dwarf temperature of $12900 \mathrm{~K}$, matching that measured by Campbell et al. (2015).

\subsection{Bootstrapping}

The flickering of the system and the variability of the white dwarf discussed may induce systematic errors in the light-curve fits. This error is not taken into account in our fit to the phase-folded data. In order to estimate the effect of these possible systematic errors on the mass ratio, we performed a bootstrap analysis. This involved selecting with replacement 44 from the 44 ULTRACAM and CHIMERA $g$ ' eclipses and 41 from the $41 r$ ' eclipses, then phase-folding and binning the light curves. The procedure was repeated 1000 times in each band. Each output light curve was converged on with an LCURVE model, using a combination of simplex and Levenberg-Marquardt algorithms. The results had a mean of $q=0.0281 \pm 0.0007$, where the uncertainty is the standard deviation of individual results.

\subsection{Contact phase measurements}

Several peculiarities of Gaia14aae make it particularly difficult to model by the method discussed above. First, the bright spot of the system is weak when compared to the flickering of the system. Therefore, an inopportune spike of flickering during the bright spot eclipse can cause the fitting routine to misfit the bright spot feature, sending the model towards inaccurate parameter values. Secondly, the ingress of the bright spot eclipse coincides with the egress of the white dwarf eclipse, causing some amount of degeneracy in fitting these features.

We therefore explored an independent method of identifying $q$, in order to test the veracity of the MCMC modelling results. We carried out a method of measuring bright spot contact phases based on that described in Wood et al. (1986). From these contact phases, a unique value of $q$ can be determined. Several assumptions, similar to those underlying the MCMC fitting method, are required for this: we assume that the bright spot is a point source, that it lies on the path of the infalling matter, and that the path of the matter can be described purely ballistically.

For each eclipse, we generated a model of best fit to the light curve. From this model, we separated the white dwarf component and subtracted it from the original data, leaving residuals that should describe just the eclipses of the bright spot and accretion disc. We then performed a numerical differentiation on these residuals. In eclipses with clear bright spot features, the bright spot ingress is clearly visible in the differentiated data as a contiguous set of negative values, and the egress as positive values. Contact points $1,2,3$, and 4 were defined as the start and end of ingress and the start and end of egress, respectively; in other words, the point at which the gradient departed from 0 or returned to 0 . Mid-ingress was defined as halfway between contact points 1 and 2, and midegress as halfway between contact points 3 and 4 . Uncertainties on each ingress and egress were judged by eye. Any eclipses without clear ingress or egress features were skipped.

Several caveats must be stated regarding this method (see e.g. Feline et al. 2004). First, it is vulnerable to the same systematic biases as the MCMC method regarding flickering and the coincident 


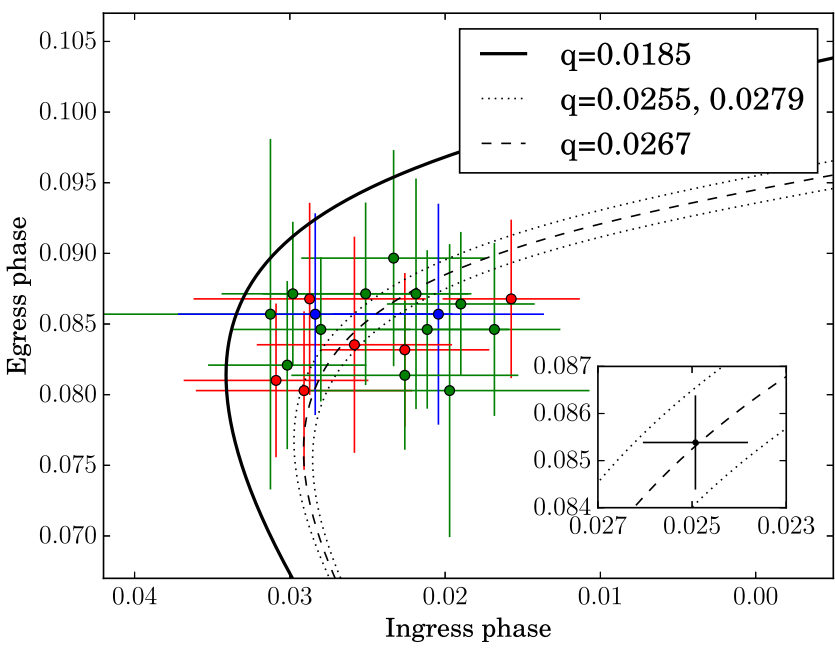

Figure 6. Bright spot ingress and egress phases measured by eye from the differential of individual ULTRACAM eclipse light curves, using the method described in Section 4.3. These measurements were made for $u$ (blue), $g^{\prime}$ (green), and $r^{\prime}$ (red) eclipses. Also shown are ballistic stream paths in ingress/egress space for the $q$ values stated. In this manner, $q$ can be estimated, under the assumption that the bright spot is a point source that lies on the ballistic stream path. The value $q=0.0185$ is the minimum mass ratio possible given the phase width of the white dwarf eclipse. Inset: the weighted mean of these measurements, compared to the same ballistic streams.

white dwarf egress. Our hope is that the human eye might be better at identifying these features correctly than the automated technique, but this is far from certain. Secondly, it is subject to a human bias in that the observer looks for eclipse features where they are expected to be. We therefore use this method primarily as a check on the reasonableness of the MCMC results, rather than as an alternative method.

With the aforementioned caveats in place, the measured bright spot mid-ingress and mid-egress phases are shown in Fig. 6. There is a significant scatter in the results, which seems to be well described by the estimated uncertainties. All measurements imply $q>0.0185$, the minimum $q$ predicted from the phase width of the white dwarf eclipse (see Section 5). The $1 / \sigma$ weighted mean of these measurements is plotted in Fig. 6 (inset), along with

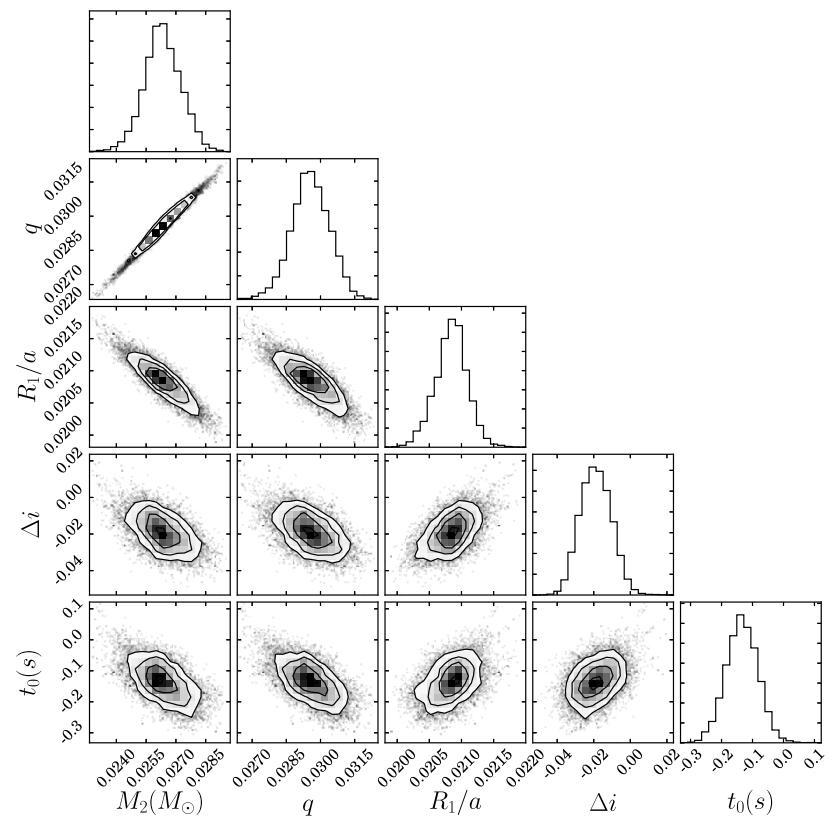

Figure 7. Corner plot of key parameters in the MCMC fit to the phasefolded $r$ ' data. We also include $M_{2}$, which is not itself a parameter of the fit, but is derived from $q$ and $R_{1}$.

uncertainties propagated from the error bars on individual measurements. These mean ingress and egress values and their uncertainties correspond to a value of $q=0.0267 \pm 0.0012$. This will be compared to the MCMC results given in Section 5.3.

\section{MODELLING RESULTS AND DISCUSSION}

The results of fits to individual eclipses are summarized in Table A1. The best-fitting models to the phase-folded $g$ ' and $r$ ' light curves are shown in Fig. 5, and their parameters presented in Table 3. We show as an example the corner plot of key fit parameters to the folded $r$ ' data in Fig. 7.

Table 3 also includes several observables that describe the eclipses: the phase width of the white dwarf eclipse from midingress to mid-egress $(\Delta \phi)$, the duration of the white dwarf ingress or egress $(\Delta w)$, and the ingress and egress phases for the bright spot

Table 3. Summary of the modelling results based on the phase-folded data. The means given in the final column here are produced using a weighting of $1 / \sigma^{2}$. Uncertainties are the formal MCMC uncertainties except where marked.

\begin{tabular}{lccc}
\hline Parameters & $r^{\prime}$ & $g^{\prime}$ & Mean \\
\hline$\Delta \phi$ & $0.0373 \pm 0.0005$ & $0.0373 \pm 0.0004$ & $0.0373 \pm 0.0003$ \\
$\Delta w$ & $0.00787 \pm 0.00014$ & $0.00800 \pm 0.00011$ & $0.00795 \pm 0.00009$ \\
$\phi_{\text {spot, } \mathrm{i}}$ & $0.0220 \pm 0.0007$ & $0.0234 \pm 0.0005$ & $0.0229 \pm 0.0004$ \\
$\phi_{\text {spot } \mathrm{e}}$ & $0.0863 \pm 0.0004$ & $0.0845 \pm 0.0005$ & $0.0856 \pm 0.0003$ \\
$q$ & $0.0283 \pm 0.0007$ & $0.0290 \pm 0.0006$ & $0.0287 \pm 0.0020^{*}$ \\
$i\left({ }^{\circ}\right)$ & $86.40 \pm 0.12$ & $86.27 \pm 0.10$ & $86.3 \pm 0.3^{*}$ \\
$M_{1}\left(\mathrm{M}_{\odot}\right)$ & $0.870 \pm 0.007$ & $0.872 \pm 0.007$ & $0.87 \pm 0.02^{*}$ \\
$M_{2}\left(\mathrm{M}_{\odot}\right)$ & $0.0246 \pm 0.0008$ & $0.0253 \pm 0.0007$ & $0.0250 \pm 0.0013^{*}$ \\
$R_{1} / a$ & $0.0215 \pm 0.0002$ & $0.0215 \pm 0.0002$ & $0.0215 \pm 0.0006^{*}$ \\
$R_{2} / a$ & $0.1393 \pm 0.0011$ & $0.1404 \pm 0.0009$ & $0.140 \pm 0.002^{*}$ \\
$R_{\text {disc }} / a$ & $0.570 \pm 0.008$ & $0.640 \pm 0.006$ & $0.615 \pm 0.005$ \\
$R_{\text {spot }} / a$ & $0.442 \pm 0.004$ & $0.421 \pm 0.005$ & $0.434 \pm 0.003$ \\
$a\left(R_{\odot}\right)$ & $0.430 \pm 0.001$ & $0.430 \pm 0.001$ & $0.430 \pm 0.003 *$ \\
\end{tabular}

* On the starred results in the final column, error bars are inflated to include the estimated systematic uncertainty in $q$, as discussed in Section 5.3. 


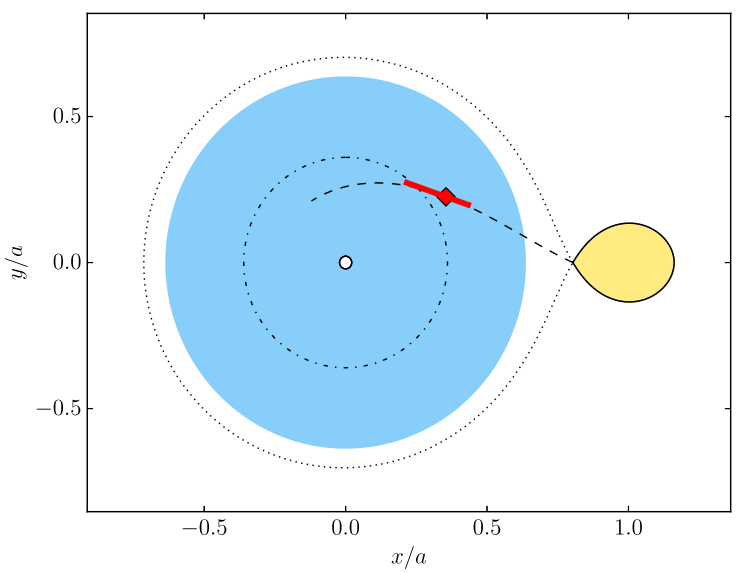

Figure 8. Schematic geometry of Gaia14aae calculated from the best-fitting model to the phase-folded $r$ ' data, showing the central white dwarf (white), the accretion disc (blue), and the donor star (yellow). The position of the bright spot peak luminosity is shown by the red diamond, with the red line showing the extension to half-maximum of the bright spot. The path of the infalling stream is shown as a dashed line, the edge of the primary Roche lobe as a dotted line, and the circularization radius as a dashed-dotted line.

eclipse $\left(\phi_{\text {spot, } \mathrm{i}}, \phi_{\text {spot, e }}\right)$. These parameters are calculated from the best-fitting models.

The geometry of the best-fitting model for the $r$ ' phase-folded data is shown in Fig. 8. The geometry of the disc and bright spot are particularly worthy of note. We find an accretion disc radius very close to the Roche lobe radius, and larger than the theoretical tidal limit of 0.58 a (Paczyński 1977, but note this approximation assumes no viscosity, and begins to deviate from the true limit for $q<0.03$ ). The bright spot is not located on the edge of the disc, but closer to the circularization radius. However, this is dependent on the modelled brightness profile of the disc, which may not accurately represent the physical disc. The bright spot in our best-fitting model also appears to be significantly extended along the direction of the infalling stream.

\subsection{Ephemeris}

From the mid-eclipse times, $t_{0}$, for individual eclipses listed in Table A1, we calculated an ephemeris for Gaia14aae of

$\operatorname{BMJD}(\mathrm{TDB})=57153.6890966(4)+0.03451957084(8) E$

where $E$ is the cycle number and the time of zero phase corresponds to the centre of the white dwarf eclipse. The quoted zero phase was chosen so as to minimize correlation between the zero-point and period, and the quoted uncertainties are $1 \sigma$. The O-C diagram for this ephemeris is shown in Fig. 9.

This ephemeris differs significantly from that quoted in Campbell et al. (2015). This difference arises from an $11 \mathrm{~s}$ discrepancy in the Bologna Faint Object Spectrograph and Camera data used as part of that ephemeris calculation, possibly a systematic offset in the instrument which is not built for precise timings. Due to this and the relatively low time resolution of the light curves used by Campbell et al. (2015), we did not include their data in our ephemeris calculation.

Including all data in individual colour bands, we have 119 measured eclipse times, giving us 117 degrees of freedom. The $\chi^{2}$ of this linear ephemeris is 105.2.

A linear ephemeris agrees with expectations. The period growth of this system is expected to be dominated by angular momentum loss due to gravitational wave radiation. Using the Landau \& Lifshitz (1971) formula

$$
\left(\frac{\dot{J}}{J}\right)=-\frac{32}{5} \frac{G^{3}}{c^{5}} \frac{M_{1} M_{2}\left(M_{1}+M_{2}\right)}{a^{4}}
$$

we would expect $\dot{J} / J=-1.6 \times 10^{-17} \mathrm{~s}^{-1}$. Under the assumptions that mass transfer is conservative and that angular momentum loss is completely dominated by gravitational wave radiation, $\dot{P}_{\text {orb }}$ can then be found (Deloye et al. 2007) by

$$
\left(\frac{\dot{P}_{\text {orb }}}{P_{\text {orb }}}\right)=3\left(\frac{\dot{J}}{J}\right)\left[\frac{\xi_{R_{2}}-1 / 3}{\xi_{R_{2}}+5 / 3-2 q}\right]
$$

where $\xi_{R_{2}}=\mathrm{d}\left(\log R_{2}\right) / \mathrm{d}\left(\log M_{2}\right)$. Taking $\xi_{R_{2}} \approx-0.2$ as the approximate gradient of the $M-R$ tracks in Fig. 13 gives $\dot{P}_{\text {orb }} \approx$ $1.7 \times 10^{-6} \mathrm{~s} \mathrm{yr}^{-1}$. Given these data, a quadratic term added to the ephemeris in equation (1) would not be detected to $3 \sigma$ unless its value were $\gtrsim 9 \times 10^{-6} \mathrm{~s} \mathrm{yr}^{-1}$. The predicted change is therefore not detectable with these data. However, as the detectability scales with $t^{2}$, and our current baseline is only two years, we can expect the period change to become detectable within the next few years.

Copperwheat et al. (2011) found some evidence of departures from a linear ephemeris in the other eclipsing AMCVn type binary, YZLMi, which were ascribed to systematic errors induced by flickering or the superhump period. To search for similar systematic biases in Gaia14aae, we checked for a correlation between the central times of each eclipse and the brightness of the disc as measured during that eclipse. We find no strong correlation, but there are two eclipses which have both an unusually bright disc and an unusually late eclipse time. Both are $i$ ' measurements, out of only three eclipses which were measured in $i$ '. This is the band in which the disc is brightest. It is therefore possible that the $i$ ' data may be biased in some way by the disc eclipse. In $u^{\prime}, g^{\prime}$, and $r^{\prime}$, we find no evidence for a similar correlation.

We searched for significant periods in a Lomb-Scargle periodogram (Lomb 1976; Scargle 1982) of these data outside of eclipse. Other than the orbital period and its harmonics, we found no significant periods. By comparing with injected, sinusoidal signals, we estimate that we would detect a signal of approximately 1 per cent strength or greater. After subtracting a sinusoid of period equal to the orbital period from the data and creating another LombScargle transform from the residuals, we still do not detect any other periods. Note that, given the white dwarf temperature of $12900 \mathrm{~K}$ (Campbell et al. 2015), we do not expect to see DBV pulsations.

\subsection{White dwarf luminosity and colour}

For each eclipse, we measured the flux contribution from the white dwarf in our best-fitting model and calculated the $u^{\prime}-g^{\prime}$ and $g^{\prime}-r$ ' colour indices. We calculated these separately for 2015 January and May/June, as well as the $g^{\prime}-r$ ' colour from 2016 August. These are shown in Fig. 10. These colours are corrected for interstellar extinction according to Schlegel, Finkbeiner \& Davis (1998) and Schlafly \& Finkbeiner (2011), using $E(B-V)=0.018$. We also show for comparison the expected colours from DB and DA white dwarf atmosphere models (Holberg \& Bergeron 2006; Kowalski \& Saumon 2006; Tremblay et al. 2011; Bergeron et al. 2011). Our colours are approximately $2 \sigma$ different from those expected for any DB white dwarf, although the closest model at $13000 \mathrm{~K}$ is similar to the expected temperature of $12900 \mathrm{~K}$, based on its GALEX ultraviolet flux (Campbell et al. 2015). It should be noted that the white dwarf may not have a pure DB atmosphere; the accreted material may have a significant fraction of heavier elements, particularly 


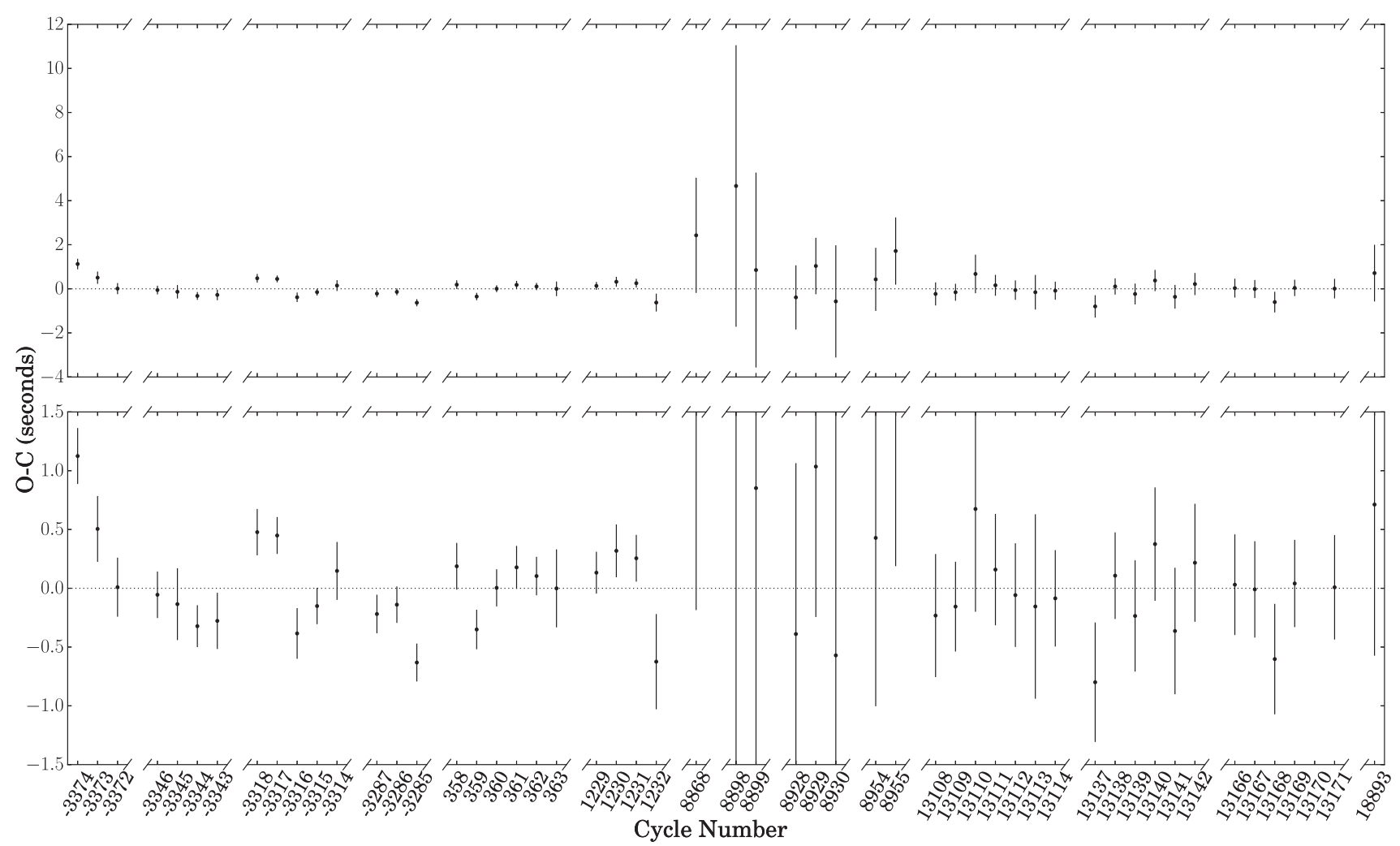

Figure 9. Mid-eclipse timing measurements of Gaia14aae, showing the difference between the measured times and those predicted from the ephemeris in equation (1). The bottom panel is a zoomed-in version of the top. Measurements in multiple colour bands have been combined using a weighted mean for this diagram, although these were treated as separate eclipses for the calculation of the ephemeris.

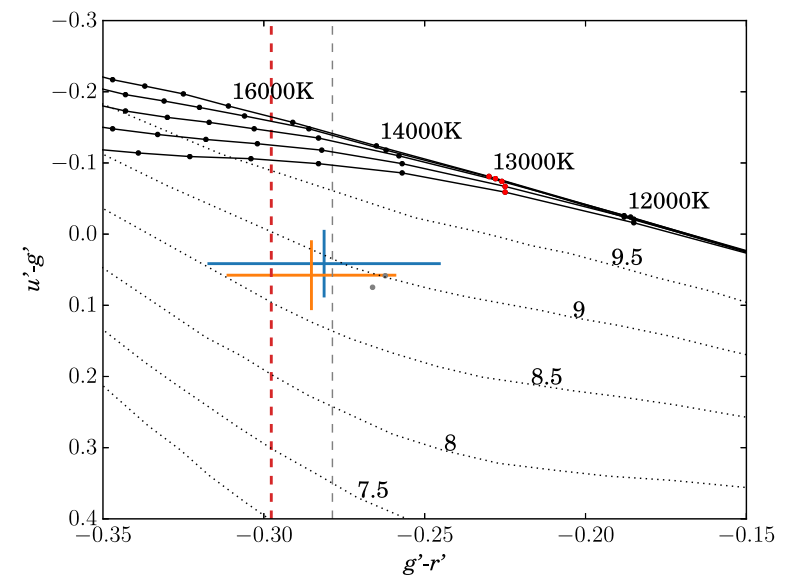

Figure 10. The position of the primary white dwarf in colour-colour space, as measured in 2015 January (cyan), 2015 May/June (orange), and 2016 August (red dashed line, $g^{\prime}-r^{\prime}$ constraint only). These fluxes have been corrected for reddening. Grey points show the positions of these measurements prior to reddening correction. Also shown are DB model atmospheres (red dots $13000 \mathrm{~K}$ models, black dots other temperatures, and solid lines connecting DB models of constant surface gravity) and DA model atmospheres (dotted lines for models of constant surface gravity), with temperatures and surface gravities labelled (Holberg \& Bergeron 2006; Kowalski \& Saumon 2006; Tremblay, Bergeron \& Gianninas 2011; Bergeron et al. 2011).
$\mathrm{C}, \mathrm{N}, \mathrm{O}$, and $\mathrm{Ne}$, depending on the prior evolution of the donor (Yungelson 2008; Nelemans et al. 2010). The white dwarf lies in a region of colour space that is occupied by some DA white dwarfs, but its spectrum shows no sign of hydrogen and a DA white dwarf of this colour would require a higher temperature. If the white dwarf eclipse depths are indeed biased by some region of the disc as discussed below, this could be evidence of a colour dependence to that bias.

By comparing the $g$ ' flux of the central white dwarf to that predicted for a DB white dwarf with a temperature of $13000 \mathrm{~K}$ and $\log (g)$ of 8.5 (Holberg \& Bergeron 2006; Bergeron et al. 2011), we estimate a distance of $188 \pm 13 \mathrm{pc}$. Of course, if there is some obscuration of the white dwarf by the disc, this estimate may not be reliable. The parallax that will be measured by Gaia will provide a more reliable estimate of the distance to this system.

The flux of the white dwarf over time is shown in Fig. 11. Between 2015 January and 2016 August, we measured a decrease of $(26 \pm 2)$ per cent in both $r$ ' and $g$ ' bands. The ratio between $r$ ' and $g$ ' stayed approximately constant.

This could be due to genuine cooling of the white dwarf, which would have been heated by its outbursts in 2014. Assuming the white dwarf is approximately described by a blackbody spectrum at $13000 \mathrm{~K}$, and treating the filters as top-hat functions whose value is 1 inside their full width at half-maximum and 0 elsewhere, this flux decrease would imply a temperature change of $\gtrsim 1000 \mathrm{~K}$ over the 18 month time interval (beginning approximately $150 \mathrm{~d}$ after the most recent outburst). This change is slightly less than the cooling seen in the short-period CV WZ Sge over the period 150-700 d after 


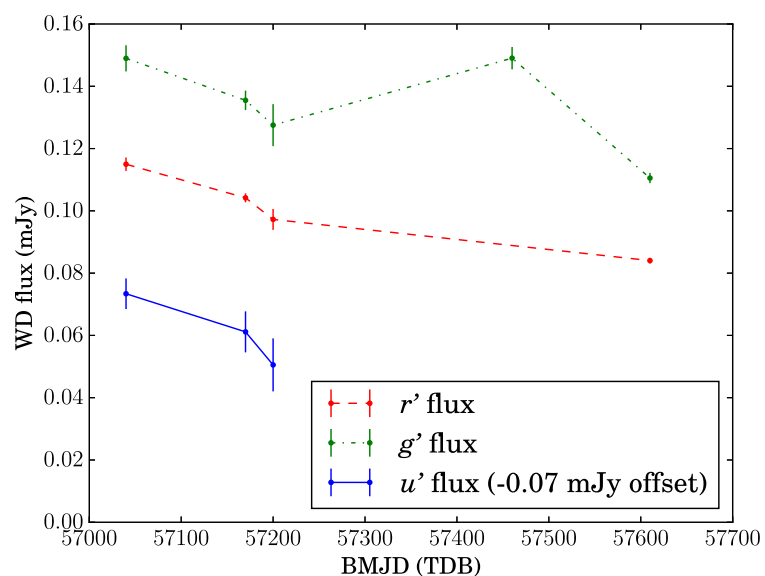

Figure 11. Evolution of the measured white dwarf flux over time, averaged between eclipse depth measurements from individual eclipses. These fluxes have been corrected for reddening. The g' measurement from 2016 March (MJD 57500) is possibly biased by the large amount of flickering during these observations. The most recent outburst for this system occurred prior to the start of observations, at MJD $=56880$

its outburst (Godon et al. 2004), and less than seen in GW Lib over the period 3-4 yr after its outburst (Szkody et al. 2012). Such a temperature change would not produce a significant colour change, which is consistent with our observations.

It should be noted that the $12900 \mathrm{~K}$ measurement of Campbell et al. (2015) is higher than the $\approx 11000 \mathrm{~K}$ temperature prediction of Bildsten et al. (2006), which was based on compressional heating of the white dwarf by the accreted matter. Bildsten et al. (2006) predict that the temperature of the central white dwarf is driven by accretion-induced heating until the system reaches an orbital period of around $40 \mathrm{~min}$. For periods longer than this the decreased mass accretion rate means that the temperature decouples from accretion-driven heating, and the white dwarf then follows standard cooling tracks. However, as will be discussed in the following sections, the donor star in Gaia14aae seems to have a higher mass and radius than was assumed by Bildsten et al. (2006), resulting in a higher mass transfer rate, which may explain this elevated temperature.

While a change in the temperature of the central white dwarf is one possible explanation of the change in eclipse depth, other possibilities must be considered. This change in eclipse depth could be explained if there were a component of the system which is not included in our models and which is not resolved from the white dwarf eclipse, such that the apparent dimming of the white dwarf is in fact the dimming of this other component. Wood et al. (1986) and Spark \& O'Donoghue (2015) discussed the optical visibility of the 'boundary layer' through which the white dwarf accretes from its disc. Though this boundary layer can theoretically be resolved from the white dwarf by the shape of the eclipse ingress and egress, doing so would require a higher signal-to-noise ratio than is currently available for this system. If present, a bright equatorial boundary layer might bias measurements of the white dwarf radius towards smaller or larger values, and a variable boundary layer might therefore induce a correlation between the modelled white dwarf luminosity and radius. We checked for such a correlation in our best-fitting models to individual eclipses, as shown in Fig. 12. We did not find a significant correlation.

We do find a reasonably strong correlation between the temperature of the white dwarf and that of the disc. This could be evidence that some disc luminosity is not resolved from the white dwarf eclipse, biasing our measurement of white dwarf luminosity. Alternatively, there may be some causal link between the two, if both are heated by accretion or if both are still decreasing following the 2014 outburst.

The measurement of $q$ described in the next section is constrained by the contact phases of white dwarf and bright spot eclipses. Therefore, $q$ should not be affected by a bias in the apparent depth of the white dwarf eclipse. However, if the same mechanism were to bias the phase width of the white dwarf eclipse this would be a problem. We therefore searched for a correlation between the depth and phase width of the white dwarf eclipse as measured from our model fits, but found no evidence for a significant correlation (see Fig. 12).

\subsection{Mass ratio and donor mass}

From the phase width of the white dwarf eclipse, a lower limit on the mass ratio can be found by assuming $i=90^{\circ}$. The phase width shown in Table 3 gives $q_{\text {min }}=0.0185 \pm 0.0005$. This is comparable to the lower limit found by Campbell et al. (2015), $q_{\min }=0.019$.

Thus far in this paper, we have described several methods of determining $q$ : MCMC fitting to phase-folded light curves and to light curves of individual eclipses, fitting to a collection of bootstrapped phase-folded light curves, and measurement of bright spot contact
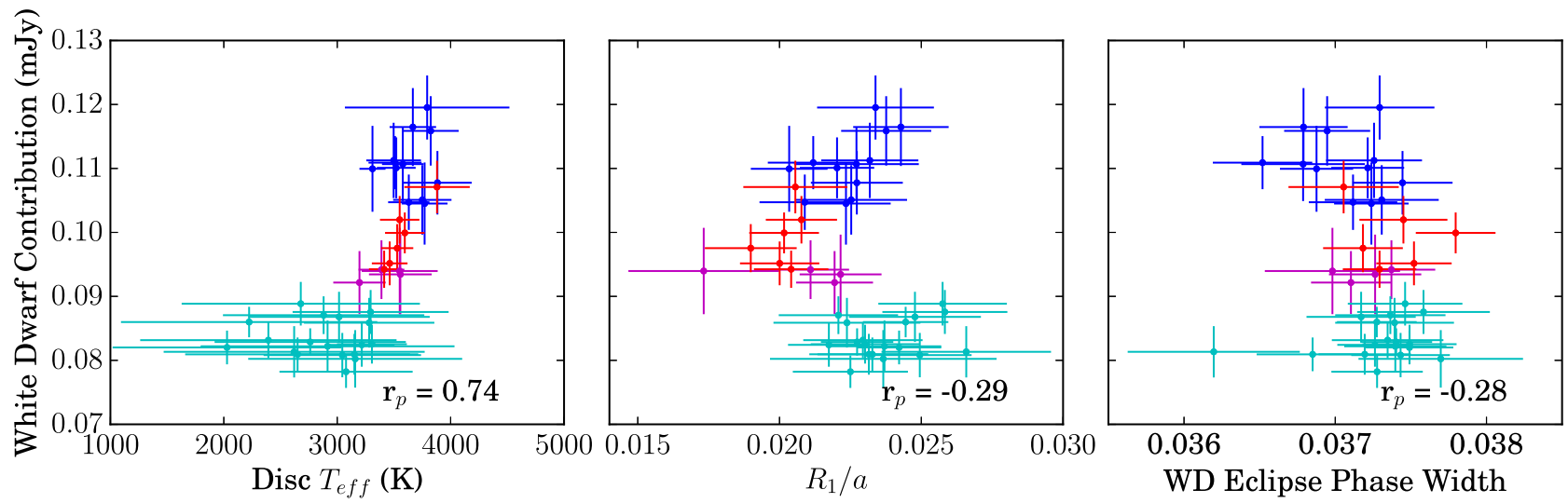

Figure 12. White dwarf luminosity as a function of other fit parameters across all ULTRACAM and CHIMERA $r$ '-band eclipses. The correlation coefficient quoted is Pearson's $r$. Colours represent the dates on which the data were taken: 2015 January (blue), 2015 May (red), 2015 June (magenta), and 2016 August (cyan). 
Table 4. Summary of the $q$ values found by the different methods described in Section 4.

\begin{tabular}{lc}
\hline Method & $q$ \\
\hline Phase-folded light curves MCMC & $0.0287 \pm 0.0005$ \\
Individual light curves MCMC & $0.030 \pm 0.003$ \\
Bootstrapping method & $0.0281 \pm 0.0007$ \\
Contact phase measurement & $0.0267 \pm 0.0012$
\end{tabular}

phases by hand. The results found by each of these methods are summarized in Table 4.

The most rigorous method for calculating $q$ is the phase-folded MCMC method, and hence we favour the result from this method. However, the scatter of the results in Table 4 suggests that the formal uncertainty on $q$ quoted by the MCMC may be an underestimate. This is not unexpected, as the method does not take into account any systematic biases that may come from the variability of the source between eclipses. We therefore suggest a more conservative error bar of 0.0020 , in an attempt to take this scatter into account. Our canonical mass ratio is then $q=0.0287 \pm 0.0020$.

To find the corresponding uncertainties on other stellar parameters, most importantly $M_{2}$, we propagated this uncertainty through the derivation of the other parameters from the raw observables $\Delta \phi$ and $\Delta w$. The resulting uncertainties are shown in the marked entries in Table 3.

\subsection{Comparison to models}

The evolutionary history of Gaia14aae can be explored by comparing the mass of the donor star with theoretical evolutionary tracks. From Kepler's third law and the constraint that the radius of the donor star equals the radius of the Roche lobe come the relation between donor mass and radius for a given orbital period (Faulkner, Flannery \& Warner 1972)

$$
P_{\text {orb }}=101 \mathrm{~s}\left(\frac{R_{2}}{0.01 R_{\odot}}\right)^{\frac{3}{2}}\left(\frac{0.1 \mathrm{M}_{\odot}}{M_{2}}\right)^{\frac{1}{2}} .
$$

This can alternately be expressed as a constraint on mean donor density coming from the orbital period alone. Therefore, our measurement of $M_{2}$ combined with the orbital period of Gaia14aae ( $P_{\text {orb }}=49.71 \mathrm{~min}$ ) can locate the donor in $M-R$ space, allowing us to compare the donor properties with the evolutionary tracks.

We take numerically calculated $M-R$ evolution tracks for AMCVn donors that have been published for the white dwarf donor (Deloye et al. 2007), helium-star donor (Yungelson 2008), and evolved-CV (Goliasch \& Nelson 2015) formation channels. We show these tracks in Fig. 13.

We show white dwarf donor models with total binary mass $M_{\text {tot }}=0.825 \mathrm{M}_{\odot}$, similar to our measured $M_{\text {tot }} \approx 0.91 \mathrm{M}_{\odot}($ Deloye et al. 2007). These tracks are for a range of initial donor degeneracies, with the top tracks being the least degenerate. The highest of these denotes the approximate boundary between the least degenerate white dwarf donor tracks and the most degenerate helium-star donor tracks. The helium-star donor tracks are for a binary with initial conditions $M_{1, \mathrm{i}}=0.8 \mathrm{M}_{\odot}, M_{2, \mathrm{i}}=0.65 \mathrm{M}_{\odot}$ and are distinguished by the evolutionary status of the donor, with the top tracks being the least evolved and the bottom tracks being the most evolved (Yungelson 2008).

For the white dwarf and helium-star evolution tracks, a donor would evolve into contact at the right-hand side of Fig. 13 and evolve through mass transfer to a lower mass and longer orbital period (moving right to left in Fig. 13). At a period of $\approx 40 \mathrm{~min}$, both Deloye et al. (2007) and Yungelson (2008) predict that the thermal time-scale of the donor becomes shorter than the mass-loss time-scale, allowing the donor to cool in response to mass loss. The donor therefore loses entropy and contracts towards complete degeneracy. For both evolutionary channels, donors with orbital periods significantly longer than this are expected to be completely degenerate.

Our measured mass and radius lie outside the predicted parameter space for the white dwarf donor evolutionary channel. Given the density constraint arising from its period, the true donor mass would have to be a factor of 2-3 times smaller to agree with the tracks shown here. Indeed, the density constraint coupled with the minimum donor mass found by Campbell et al. (2015) are sufficient to disagree with the predicted tracks, including the collapse due to cooling, without needing the mass estimate presented in this work. Irradiation of the donor can delay the predicted collapse to longer periods. Even so, our mass estimate would lie significantly above the least degenerate white dwarf donor track. We therefore find that Gaia14aae is unlikely to have evolved through the white dwarf donor track.

The helium-star channel evolutionary tracks calculated by Yungelson (2008) end once the AMCVns reach periods of around $40 \mathrm{~min}$, making it difficult to compare our measured mass and radius to these tracks. The predicted collapse towards degeneracy beyond this point (the beginning of which is just visible at the left-hand end of these tracks) would disagree with our measurements. However, if this collapse were delayed to a period of $50 \mathrm{~min}$ or longer, our measurements would appear to be close to agreement with the least degenerate of the tracks shown here. Delaying this collapse to longer periods may be possible by, for instance, increasing the efficiency of irradiation of the donor by the accretor (Deloye et al. 2007) or by outbursts.

We also show two evolved-CV tracks. The first, from Goliasch \& Nelson (2015, dashed-dotted line), has a $0.6 \mathrm{M}_{\odot}$ accretor, $\dot{M}=1.4 \times 10^{-11} \mathrm{M}_{\odot} \mathrm{yr}^{-1}$, and a hydrogen fraction of $\sim 5$ per cent in the transferred gas. The second track (Goliasch \& Nelson, private communication, dotted line) has an accretor of $0.85 \mathrm{M}_{\odot}$. These tracks are roughly representative of the edge of region of $M-R$ space this channel explores; systems above and to the left of these tracks (including Gaia14aae) could easily have formed by this channel. However, AMCVns that form by this channel are expected to retain a greater fraction of their hydrogen than AM CVns that form by the other two channels. Evolved-CV tracks with a smaller hydrogen fraction than $\sim 1$ per cent in this region of $M-R$ space are possible, but they can only form from progenitor CVs with a limited range of initial parameters, and require long time-scales to evolve. We might therefore expect to see a number of short-period CVs with visible hydrogen and helium for each AM CVn that forms by this channel. At present few such CVs are known (see Augusteijn et al. 1996; Breedt et al. 2012, for examples).

In terms of its measured mass and radius, the donor in Gaia14aae appears to fit best with tracks of the evolved-CV channel. However, the predicted hydrogen content of systems formed by these tracks is not seen in spectra of Gaia14aae (Campbell et al. 2015). Though no quantitative upper limit exists for Gaia14aae, a hydrogen abundance on the order predicted for this channel would be easily detectable: modelling of the AMCVn systems GPCom (with local thermal equilibrium models, Marsh, Horne \& Rosen 1991) and V396 Hya (with non-local thermal equilibrium models, Nagel, Rauch \& Werner 2009) find that any hydrogen abundances of 


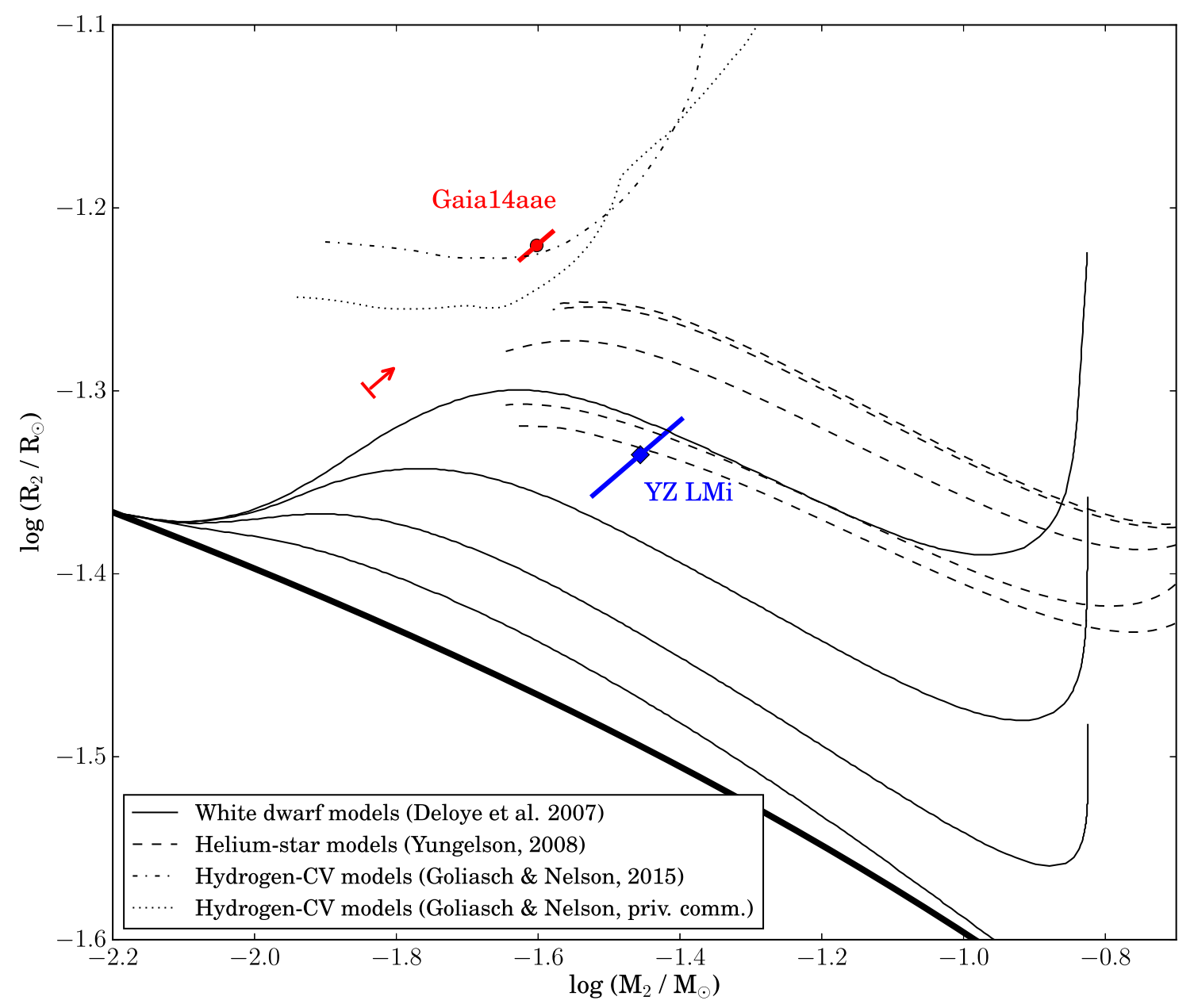

Figure 13. The measured donor masses and radii of Gaia14aae (red circle) and J0926 (blue diamond) and their uncertainties (solid lines of corresponding colours) compared to evolutionary tracks from Deloye et al. (2007, thin black lines), Yungelson (2008, dashed), Goliasch \& Nelson (2015, dashed-dotted), and Goliasch \& Nelson (private communication, dotted). The white dwarf and helium-star donor tracks include multiple levels of degeneracy, with the most degenerate models at the bottom. We also show the $M-R$ track for a zero-entropy donor (thick solid line). The arrow shows the lower limit on $M_{2}$ corresponding to $q_{\min }=0.0185$ for Gaia14aae. The diagonal uncertainties result from the strong constraints on the donors' mean densities resulting (according to equation 4 ) from their tightly constrained orbital periods.

$\gtrsim 10^{-5}$ should result in detectable Balmer emission. Similar upper limits have been found for DB white dwarfs in this temperature range (Koester \& Kepler 2015; Bergeron et al. 2011). Producing similar tracks without detectable hydrogen would require finely tuned starting parameters that make them unlikely (Goliasch \& Nelson, private communication). The absence of any visible hydrogen in the optical spectrum of Gaia14aae is therefore difficult to reconcile with predictions for this channel.

We conclude that Gaia14aae may have formed by either the evolved-CV channel or the helium-star donor channel. The results presented here are not in perfect agreement with either of the models as they currently stand, but one or both of these channels may have a region of parameter space that can explain the properties of Gaia14aae. The close agreement of our measured $M_{2}$ with that of the evolved-CV channel is particularly intriguing, given that this channel has generally been considered the least probable formation channel. The clear disagreement of our result with the white dwarf donor formation channel, which has been widely considered to be the dominant channel, is also of interest in light of the prediction by Shen (2015) that double white dwarf binaries will not reach a state of stable accretion.

\subsection{Implications for gravitational wave emission}

The distribution of AMCVn-originated gravitational wave strains detectable by the Laser Interferometer Space Antenna (LISA) depends on the distribution of both periods and stellar masses across AMCVns. The donor mass of Gaia14aae presented above is greater than would be expected for a fully degenerate donor in a binary at this orbital period. Consequently, the gravitational wave emission will be greater.

Due to the high inclination of Gaia14aae, the $h_{+}$component of its gravitational wave emission will dominate over the $h_{\times}$component. The amplitude of the $h_{+}$component can be calculated (Korol et al. 2017) by

$h_{+}=\frac{2(G \mathcal{M})^{5 / 3}\left(2 \pi / P_{\text {orb }}\right)^{2 / 3}}{c^{4} d}\left(1+\cos ^{2} i\right)$

where $\mathcal{M}=\left(M_{1} M_{2}\right)^{3 / 5}\left(M_{1}+M_{2}\right)^{-1 / 5}$ is the chirp mass of the system and $d$ is its distance from the Earth. For the stellar masses shown above, we predict the strain from Gaia14aae to be

$h_{+}=1.2 \times 10^{-20} \times \frac{1 \mathrm{pc}}{d}$. 
For the distance of $188 \mathrm{pc}$, we estimated in Section 5.2, this would give a strain of $6.1 \times 10^{-23}$. This is below the detection limit of LISA, unsurprisingly given the long orbital period of Gaia14aae. $\mathrm{AMCVns}$ at the short end of their period distribution are expected to be the brightest emitters of gravitational waves.

For comparison, we consider a degenerate donor with the same orbital period but a mass of $M_{2}=0.01 \mathrm{M}_{\odot}$. The strain for that system would be $h_{+}=2.3 \times 10^{-23}$. The gravitational wave emission of Gaia14aae is therefore a factor of 2.7 higher than would be expected in the degenerate case, and the volume of space in which the system would be detectable is increased by nearly a factor of 20 . This emphasizes the need to understand the nature of AM CVn donors in order to predict the distribution of strains LISA will detect, both as resolved sources and as unresolved background. The results from both Gaia14aae and YZLMi suggest that non-degenerate and partially degenerate donors are more common among AMCVns than previously believed. If this is the case, we would expect AMCVns as a population to be brighter emitters of gravitational waves for a given orbital period than previously predicted.

\section{CONCLUSIONS}

Gaia14aae is the third known eclipsing AMCVn-type binary and the first in which the central white dwarf is fully eclipsed. As such, it provides an unprecedented opportunity to measure the properties of the component stars in one of these systems and thereby constrain the system's prior evolution. The results are difficult to reconcile with existing models.

Any measurement of the properties of Gaia14aae is complicated by several unfortunate peculiarities of the system. In particular, the weakness of a key feature of the system (its 'bright spot') means that light-curve fitting can be biased by the intrinsic red noise of the system. These difficulties increase the systematic uncertainty in our measurements. We have attempted to take this uncertainty into account by inflating the quoted error bars on our results.

We measured a mass ratio $q=0.0287 \pm 0.0020$ and a donor mass $M_{2}=0.0250 \pm 0.0013 \mathrm{M}_{\odot}$. Combined with the donor density constraint arising from the orbital period, this mass shows that the donor is not degenerate and that the system did not evolve from a double degenerate binary. The system therefore must either have a non-degenerate helium star as its donor or be descended from a hydrogen $\mathrm{CV}$ with an evolved donor. In both cases, there are unexplained questions: the donor in the former is expected to have collapsed towards degeneracy before reaching this orbital period, and the latter is expected to show traces of spectroscopic hydrogen. Neither of these predictions are observed, but it may be possible to tweak the models in order to explain Gaia14aae's evolution.

\section{ACKNOWLEDGEMENTS}

The authors would like to thank Lorne Nelson and Lev Yungelson for exceedingly helpful discussions and insight, and the anonymous referee for their constructive feedback.

MJG acknowledges funding from an the Science and Technology Facilities Council (STFC) studentship via grant ST/N504506/1. TRM, DTHS, and EB acknowledge STFC via grants ST/L000733/1 and ST/P000495/1. SB acknowledges funding from NWO VIDI grant 639.042.218, financed by the Netherlands Organisation for Scientific Research (NWO). VSD, SPL, ULTRACAM, and ULTRASPEC are funded by STFC via consolidated grant ST/J001589. Support for this work was provided by National Aeronautics and Space Administration (NASA) through
Hubble Fellowship grant no. HST-HF2-51357.001-A, awarded by the Space Telescope Science Institute, which is operated by the Association of Universities for Research in Astronomy, Incorporated, under NASA contract NAS5-26555. Part of the research was carried out at the Jet Propulsion Laboratory, California Institute of Technology, under a contract with the National Aeronautics and Space Administration. The research leading to these results has received funding from the European Research Council under the European Union's Seventh Framework Programme (FP/20072013)/ERC Grant Agreement n. 320964 (WDTracer).

This publication made use of the packages LCURVE (Copperwheat et al. 2010), NUMPY, MATPLOTLIB, ASTROPY, SCIPY, EMCEE (ForemanMackey et al. 2013), and CORNER (Foreman-Mackey et al. 2016). DA and DB model white dwarf atmospheres for Fig. 10 were taken from http://www.astro.umontreal.ca/ bergeron/CoolingModels (last accessed 2016 June 21).

The data presented in this work were obtained using the WHT operated by the Isaac Newton Group at the Roque de los Muchachos Observatory on La Palma, the $2.4 \mathrm{~m}$ TNT operated by the National Astronomy Research Institute of Thailand at the Thai National Observatory on Doi Inthanon, and the 200-inch Hale Telescope at Palomar Observatory operated by the California Institute of Technology.

\section{REFERENCES}

Anderson S. F. et al., 2005, AJ, 130, 2230

Augusteijn T., van der Hooft F., de Jong J. A., van Paradijs J., 1996, A\&A, 311,889

Bergeron P. et al., 2011, ApJ, 737, 28

Bildsten L., Townsley D. M., Deloye C. J., Nelemans G., 2006, ApJ, 640, 466

Bildsten L., Shen K. J., Weinberg N. N., Nelemans G., 2007, ApJ, 662, L95 Breedt E., Gänsicke B. T., Marsh T. R., Steeghs D., Drake A. J., Copperwheat C. M., 2012, MNRAS, 425, 2548

Campbell H. C. et al., 2015, MNRAS, 452, 1060

Cannizzo J. K., Nelemans G., 2015, ApJ, 803, 19

Carter P. J. et al., 2013, MNRAS, 429, 2143

Carter P. J. et al., 2014, MNRAS, 439, 2848

Cook M. C., Warner B., 1984, MNRAS, 207, 705

Copperwheat C. M., Marsh T. R., Dhillon V. S., Littlefair S. P., Hickman R., Gaensicke B. T., Southworth J., 2010, MNRAS, 402, 1824

Copperwheat C. M. et al., 2011, MNRAS, 410, 1113

Deloye C. J., Taam R. E., Winisdoerffer C., Chabrier G., 2007, MNRAS, 381,525

Dhillon V. S. et al., 2007, MNRAS, 378, 825

Dhillon V. S. et al., 2014, MNRAS, 444, 4009

Faulkner J., Flannery B. P., Warner B., 1972, ApJ, 175, L79

Feline W. J., Dhillon V. S., Marsh T. R., Brinkworth C. S., 2004, MNRAS, 355,1

Foreman-Mackey D., Hogg D. W., Lang D., Goodman J., 2013, PASP, 125, 306

Foreman-Mackey D. et al., 2016, Corner.Py: Corner.Py V2.0.0. Available at: https://zenodo.org/record/53155\#.WTfidLPQTmE (last accessed 2016 June 21)

Gianninas A., Strickland B. D., Kilic M., Bergeron P., 2013, ApJ, 766, 3

Godon P., Sion E. M., Cheng F., Gaensicke B. T., Howell S., Knigge C., Sparks W. M., Starrfield S., 2004, ApJ, 602, 336

Goliasch J., Nelson L., 2015, ApJ, 809, 80

Goodman J., Weare J., 2010, Commun. Appl. Math. Comput. Sci., 5, 65

Harding L. K. et al., 2016, MNRAS, 457, 3036

Hardy L. K. et al., 2017, MNRAS, 465, 4968

Holberg J. B., Bergeron P., 2006, AJ, 132, 1221

Iben I. J., Tutukov A. V., 1987, ApJ, 313, 727

Ivanova N. et al., 2013, A\&AR, 21, 59 
Kato T., Osaki Y., 2013, PASJ, 65, 115

Koester D., Kepler S. O., 2015, A\&A, 583, A86

Korol V., Rossi E. M., Groot P. J., Nelemans G., Toonen S., Brown A. G. A., 2017, MNRAS, 470, 1894

Kotko I., Lasota J.-P., Dubus G., Hameury J.-M., 2012, A\&A, 544, A13

Kowalski P. M., Saumon D., 2006, ApJ, 651, L137

Kupfer T., Steeghs D., Groot P. J., Marsh T. R., Nelemans G., Roelofs G. H. A., 2016, MNRAS, 457, 1828

Landau L. D., Lifshitz E. M., 1971, The Classical Theory of Fields, 3rd edn. Pergamon Press, Oxford

Levitan D. et al., 2011, ApJ, 739, 68

Levitan D. et al., 2013, MNRAS, 430, 996

Levitan D. et al., 2014, ApJ, 785, 114

Littlefair S. P. et al., 2014, MNRAS, 445, 2106L

Lomb N. R., 1976, Astrophys. Space Sci., 39, 447

Marsh T. R., Horne K., Rosen S., 1991, ApJ, 366, 535

McAllister M. J. et al., 2015, MNRAS, 451, 114

McAllister M. J. et al., 2017, MNRAS, 464, 1353

Nagel T., Rauch T., Werner K., 2009, A\&A, 499, 773

Nelemans G., 2003, Class. Quantum Gravity, 20, S81

Nelemans G., Yungelson L. R., Portegies Zwart S. F., 2004, MNRAS, 349, 181

Nelemans G., Yungelson L. R., Sluys M. V. V. D., Tout C. A., 2010, MNRAS, 401, 1347

Paczyński, 1977, ApJ, 216, 822

Paczyński B., 1967, Acta Astron., 17, 287

Podsiadlowski P., Han Z., Rappaport S., 2003, MNRAS, 340, 1214

Roelofs G. H. A., Groot P. J., Nelemans G., Marsh T. R., Steeghs D., 2006, MNRAS, 371, 1231
Roelofs G. H. A., Nelemans G., Groot P. J., 2007, MNRAS, 382, 685

Savonije G. J., de Kool M., van den Heuvel E. P. J., 1986, A\&A, 155, 51

Savoury C. D. J. et al., 2011, MNRAS, 415, 2025

Scargle J. D., 1982, ApJ, 263, 835

Schlafly E. F., Finkbeiner D. P., 2011, ApJ, 737, 103

Schlegel D. J., Finkbeiner D. P., Davis M., 1998, ApJ, 500, 525

Sengar R., Tauris T. M., Langer N., Istrate A. G., 2017, MNRAS, 470, L6

Shen K. J., 2015, ApJ, 805, L6

Solheim J.-E., 2010, PASP, 122, 1133

Spark M. K., O'Donoghue D., 2015, MNRAS, 449, 175

Szkody P. et al., 2012, ApJ, 753, 158

Tremblay P.-E., Bergeron P., Gianninas A., 2011, ApJ, 730, 128

Tutukov A. V., Fedorova A., Ergma E., Yungelson L., 1985, SvA Lett., 11, 52

Verbunt F., Rappaport S., 1988, ApJ, 332, 193

Wood J., Horne K., Berriman G., Wade R., O’Donoghue D., Warner B., 1986, MNRAS, 219, 629

Wood J. H. et al., 1989, MNRAS, 239, 809

Woudt P. A. et al., 2009, ApJ, 706, 738

Yungelson L. R., 2008, Astron. Lett., 34, 620

\section{APPENDIX A: RESULTS OF INDIVIDUAL ECLIPSE MODELLING}

Table A1 shows key parameters for the model fits carried out separately on individual eclipses. 
Table A1. Results from the fits carried out to individual eclipses. $\sigma$ here is the standard deviation of MCMC chain values. We also quote the weighted mean (with its propagated error) and standard deviation of these values for each parameter.

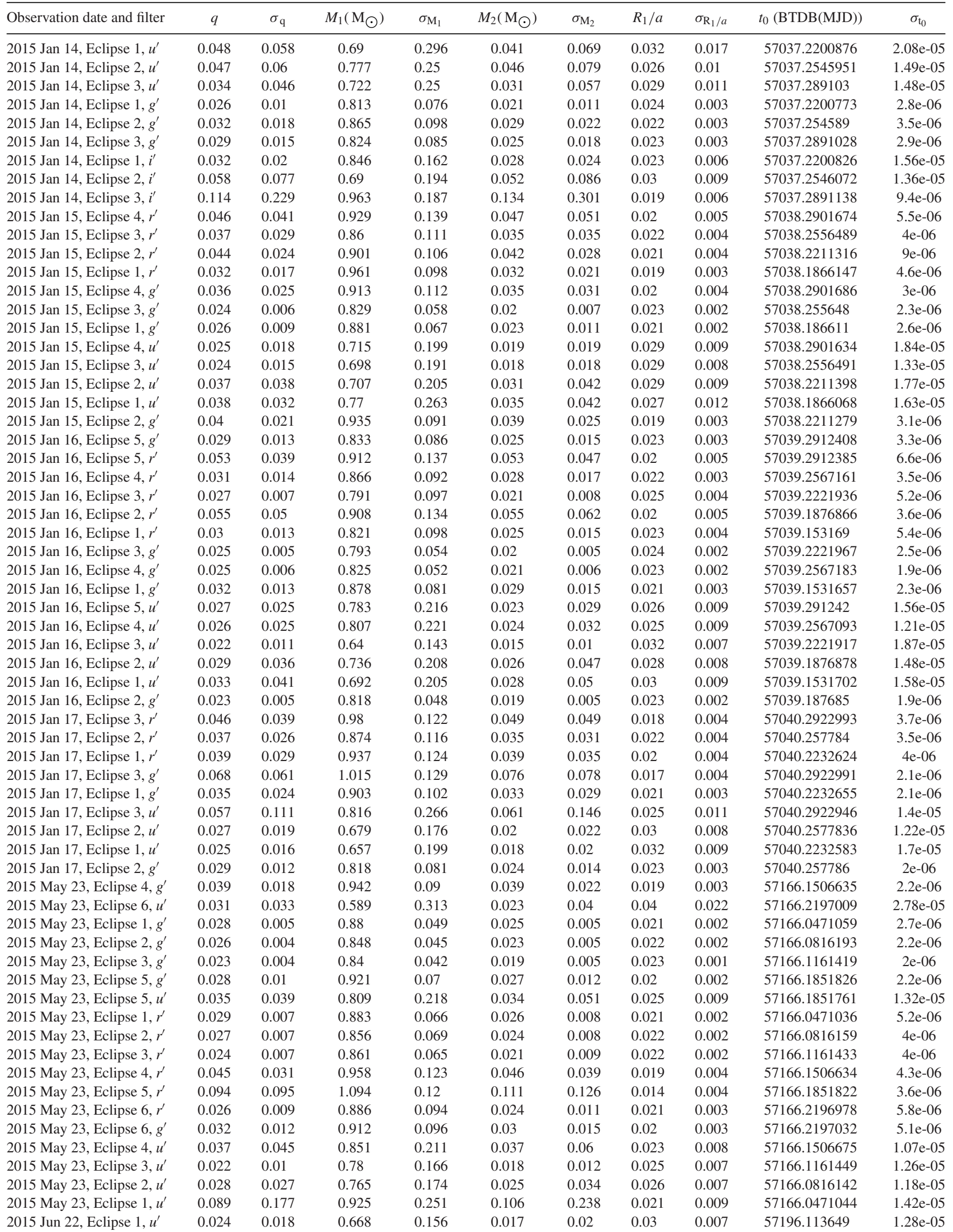


Table A1 - continued

\begin{tabular}{|c|c|c|c|c|c|c|c|c|c|c|}
\hline Observation date and filter & $q$ & $\sigma_{\mathrm{q}}$ & $M_{1}\left(\mathrm{M}_{\odot}\right)$ & $\sigma_{\mathrm{M}_{1}}$ & $M_{2}\left(\mathrm{M}_{\odot}\right)$ & $\sigma_{\mathrm{M}_{2}}$ & $R_{1} / a$ & $\sigma_{\mathrm{R}_{1} / a}$ & $t_{0}(\mathrm{BTDB}(\mathrm{MJD}))$ & $\sigma_{\mathrm{t}_{0}}$ \\
\hline 2015 Jun 22, Eclipse 4, $u^{\prime}$ & 0.044 & 0.065 & 0.408 & 0.304 & 0.029 & 0.073 & 0.059 & 0.033 & 57196.2172251 & $3.54 \mathrm{e}-05$ \\
\hline 2015 Jun 22, Eclipse 2, $g^{\prime}$ & 0.049 & 0.023 & 0.957 & 0.094 & 0.049 & 0.027 & 0.019 & 0.003 & 57196.1481697 & $2.4 \mathrm{e}-06$ \\
\hline 2015 Jun 22, Eclipse $3, u^{\prime}$ & 0.026 & 0.018 & 0.665 & 0.195 & 0.019 & 0.021 & 0.031 & 0.01 & 57196.1826902 & $1.64 \mathrm{e}-05$ \\
\hline 2015 Jun 22, Eclipse 4, $g^{\prime}$ & 0.03 & 0.016 & 0.893 & 0.115 & 0.028 & 0.019 & 0.021 & 0.004 & 57196.2172048 & $6 e-06$ \\
\hline 2015 Jun 22, Eclipse 3, $r^{\prime}$ & 0.034 & 0.02 & 0.894 & 0.099 & 0.032 & 0.025 & 0.021 & 0.003 & 57196.1826913 & $4.3 \mathrm{e}-06$ \\
\hline 2015 Jun 22, Eclipse $4, r^{\prime}$ & 0.063 & 0.093 & 1.069 & 0.153 & 0.075 & 0.127 & 0.015 & 0.005 & 57196.2171963 & $6.1 \mathrm{e}-06$ \\
\hline 2015 Jun 22, Eclipse $3, g^{\prime}$ & 0.029 & 0.009 & 0.891 & 0.069 & 0.026 & 0.01 & 0.021 & 0.002 & 57196.1826902 & $2.3 \mathrm{e}-06$ \\
\hline 2016 Mar 12, Eclipse 1, KG5 & 0.031 & 0.006 & 0.939 & 0.303 & 0.029 & 0.011 & 0.021 & 0.011 & 57459.8086767 & $3 e-05$ \\
\hline 2016 Mar 13, Eclipse 1, KG5 & 0.035 & 0.012 & 0.779 & 0.292 & 0.026 & 0.01 & 0.027 & 0.014 & 57460.8442886 & $8.1 \mathrm{e}-05$ \\
\hline 2016 Mar 13, Eclipse 2, KG5 & 0.035 & 0.017 & 0.798 & 0.365 & 0.027 & 0.014 & 0.028 & 0.017 & 57460.8787594 & $5.01 \mathrm{e}-05$ \\
\hline 2016 Aug 06, Eclipse 3, $r^{\prime}$ & 0.029 & 0.022 & 0.787 & 0.155 & 0.025 & 0.026 & 0.025 & 0.006 & 57606.2406934 & $9.7 e-06$ \\
\hline 2016 Aug 06, Eclipse 4, $r^{\prime}$ & 0.032 & 0.023 & 0.898 & 0.118 & 0.031 & 0.028 & 0.021 & 0.004 & 57606.2751919 & $5.1 \mathrm{e}-06$ \\
\hline 2016 Aug 06, Eclipse 7, $r^{\prime}$ & 0.03 & 0.022 & 0.874 & 0.118 & 0.028 & 0.028 & 0.022 & 0.004 & 57606.3787416 & $4.9 \mathrm{e}-06$ \\
\hline 2016 Aug 06, Eclipse 6, $r^{\prime}$ & 0.047 & 0.046 & 0.92 & 0.163 & 0.049 & 0.059 & 0.02 & 0.006 & 57606.3442234 & $7.8 \mathrm{e}-06$ \\
\hline 2016 Aug 06, Eclipse 2, $r^{\prime}$ & 0.024 & 0.007 & 0.83 & 0.069 & 0.021 & 0.009 & 0.023 & 0.002 & 57606.2061493 & $3.7 \mathrm{e}-06$ \\
\hline 2016 Aug 06, Eclipse 5, $r^{\prime}$ & 0.022 & 0.006 & 0.756 & 0.068 & 0.017 & 0.006 & 0.026 & 0.003 & 57606.3097068 & $5 e-06$ \\
\hline 2016 Aug 06, Eclipse 1, $r^{\prime}$ & 0.024 & 0.009 & 0.81 & 0.098 & 0.02 & 0.011 & 0.024 & 0.004 & 57606.1716347 & $5.7 \mathrm{e}-06$ \\
\hline 2016 Aug 06, Eclipse 7, $g^{\prime}$ & 0.027 & 0.015 & 0.832 & 0.094 & 0.024 & 0.018 & 0.023 & 0.003 & 57606.3787506 & $3.9 \mathrm{e}-06$ \\
\hline 2016 Aug 06, Eclipse 6, $g^{\prime}$ & 0.039 & 0.055 & 0.864 & 0.148 & 0.04 & 0.072 & 0.022 & 0.005 & 57606.344228 & $6.4 \mathrm{e}-06$ \\
\hline 2016 Aug 06, Eclipse 5, $g^{\prime}$ & 0.03 & 0.016 & 0.888 & 0.103 & 0.028 & 0.02 & 0.021 & 0.003 & 57606.3097093 & $4.1 \mathrm{e}-06$ \\
\hline 2016 Aug 06, Eclipse $4, g^{\prime}$ & 0.025 & 0.011 & 0.835 & 0.085 & 0.021 & 0.013 & 0.023 & 0.003 & 57606.2751921 & $4.2 \mathrm{e}-06$ \\
\hline 2016 Aug 06, Eclipse $3, g^{\prime}$ & 0.034 & 0.037 & 0.896 & 0.155 & 0.035 & 0.048 & 0.021 & 0.005 & 57606.2406716 & $7.6 \mathrm{e}-06$ \\
\hline 2016 Aug 07, Eclipse $2, r^{\prime}$ & 0.026 & 0.013 & 0.806 & 0.086 & 0.022 & 0.014 & 0.024 & 0.003 & 57607.2072221 & $4.3 \mathrm{e}-06$ \\
\hline 2016 Aug 07, Eclipse 4, $g^{\prime}$ & 0.033 & 0.03 & 0.873 & 0.118 & 0.032 & 0.037 & 0.022 & 0.004 & 57607.2762599 & $4.3 \mathrm{e}-06$ \\
\hline 2016 Aug 07, Eclipse 3, $g^{\prime}$ & 0.028 & 0.013 & 0.878 & 0.091 & 0.025 & 0.016 & 0.021 & 0.003 & 57607.2417351 & $4.2 \mathrm{e}-06$ \\
\hline 2016 Aug 07, Eclipse $2, g^{\prime}$ & 0.023 & 0.005 & 0.818 & 0.056 & 0.019 & 0.005 & 0.023 & 0.002 & 57607.2072187 & $2.7 \mathrm{e}-06$ \\
\hline 2016 Aug 07, Eclipse $1, g^{\prime}$ & 0.027 & 0.013 & 0.803 & 0.095 & 0.023 & 0.015 & 0.024 & 0.003 & 57607.1726871 & $4 \mathrm{e}-06$ \\
\hline 2016 Aug 07, Eclipse 5, $g^{\prime}$ & 0.028 & 0.013 & 0.867 & 0.088 & 0.025 & 0.015 & 0.022 & 0.003 & 57607.3107734 & $4 \mathrm{e}-06$ \\
\hline 2016 Aug 08, Eclipse $4, r^{\prime}$ & 0.028 & 0.016 & 0.78 & 0.1 & 0.023 & 0.014 & 0.025 & 0.004 & 57608.2773267 & $5.2 \mathrm{e}-06$ \\
\hline 2016 Aug 08, Eclipse $1, g^{\prime}$ & 0.034 & 0.021 & 0.855 & 0.107 & 0.031 & 0.025 & 0.022 & 0.004 & 57608.1737669 & $4 \mathrm{e}-06$ \\
\hline 2016 Aug 08, Eclipse $2, g^{\prime}$ & 0.027 & 0.015 & 0.837 & 0.097 & 0.024 & 0.018 & 0.023 & 0.003 & 57608.2082868 & $3 e-06$ \\
\hline 2016 Aug 08, Eclipse 3, $g^{\prime}$ & 0.027 & 0.014 & 0.802 & 0.09 & 0.023 & 0.016 & 0.024 & 0.003 & 57608.2427987 & $3 e-06$ \\
\hline 2016 Aug 08, Eclipse $4, g^{\prime}$ & 0.026 & 0.013 & 0.828 & 0.087 & 0.022 & 0.012 & 0.023 & 0.003 & 57608.2773237 & $3.1 \mathrm{e}-06$ \\
\hline 2016 Aug 08, Eclipse $6, g^{\prime}$ & 0.034 & 0.018 & 0.846 & 0.108 & 0.03 & 0.021 & 0.023 & 0.004 & 57608.3463644 & $3.4 \mathrm{e}-06$ \\
\hline 2016 Aug 08, Eclipse $1, r^{\prime}$ & 0.031 & 0.019 & 0.839 & 0.115 & 0.028 & 0.023 & 0.023 & 0.004 & 57608.1737637 & $5.6 \mathrm{e}-06$ \\
\hline 2016 Aug 08, Eclipse 2, $r^{\prime}$ & 0.033 & 0.019 & 0.878 & 0.11 & 0.03 & 0.023 & 0.021 & 0.004 & 57608.2082847 & $4.8 \mathrm{e}-06$ \\
\hline 2016 Aug 08, Eclipse 3, $r^{\prime}$ & 0.03 & 0.019 & 0.895 & 0.104 & 0.028 & 0.023 & 0.021 & 0.003 & 57608.2428044 & $3.6 \mathrm{e}-06$ \\
\hline 2016 Aug 08, Eclipse 6, $r^{\prime}$ & 0.03 & 0.014 & 0.852 & 0.1 & 0.027 & 0.016 & 0.022 & 0.003 & 57608.3463683 & $5.4 \mathrm{e}-06$ \\
\hline 2017 Feb 21, Eclipse 1, KG5 & 0.042 & 0.041 & 0.771 & 0.223 & 0.039 & 0.052 & 0.026 & 0.009 & 57805.8673572 & $1.45 \mathrm{e}-05$ \\
\hline Weighted mean & 0.030 & 0.003 & 0.808 & 0.074 & 0.025 & 0.002 & 0.022 & 0.002 & - & - \\
\hline Standard deviation & 0.014 & - & 0.092 & - & 0.017 & - & 0.005 & - & - & - \\
\hline Phase fold, $r^{\prime}$ & 0.0296 & 0.0008 & 0.87 & 0.06 & 0.026 & 0.002 & 0.021 & 0.002 & - & - \\
\hline Phase fold, $g^{\prime}$ & 0.0312 & 0.0007 & 0.90 & 0.06 & 0.028 & 0.002 & 0.021 & 0.002 & - & - \\
\hline Weighted mean & 0.0305 & 0.0005 & 0.90 & 0.04 & 0.0273 & 0.0014 & 0.0207 & 0.0014 & - & - \\
\hline
\end{tabular}

This paper has been typeset from a $\mathrm{T}_{\mathrm{E}} \mathrm{X} / \mathrm{L} \mathrm{T} \mathrm{E} \mathrm{X}$ file prepared by the author. 